\title{
Education, taxation and the perceived effects of sin good consumption
}

\author{
Giovanni Immordino ${ }^{1}$ - Anna Maria C. Menichini ${ }^{2}$ (D) Maria Grazia Romano ${ }^{2}$
}

Accepted: 18 September 2021 / Published online: 1 November 2021

(c) The Author(s) 2021

\begin{abstract}
In a setting in which an agent has a behavioral bias that causes an underestimation or an overestimation of the health consequences of sin goods consumption, the paper studies how a social planner can affect the demand of such goods through education and taxation. When only optimistic consumers are present, depending on the elasticity of demand of the sin good with respect to taxation, the two instruments can be substitutes or complements. When consumers are heterogeneous, the correcting effect that taxation has on optimistic consumers has unintended distorting effects on both pessimistic and rational ones. In this framework, educational measures, by aligning biased consumers' perceptions closer to the true probability of health damages, are more effective than taxation.
\end{abstract}

Keywords Overoptimism $\cdot$ Taxation $\cdot$ Education $\cdot$ Sin goods

JEL Classification H3 · I2

\section{Introduction}

Overoptimism is a common trait of human beings well documented in psychology and behavioral economics. An overoptimistic individual is typically inclined to think that favorable events are more likely or more positive than they actually

Anna Maria C. Menichini

amenichini@unisa.it

Giovanni Immordino

giovanni.immordino@unina.it

Maria Grazia Romano

maromano@unisa.it

1 Department of Economics and Statistics, University of Naples Federico II and CSEF, Via Cintia Monte Sant'Angelo, 80122 Naples, Italy

2 Department of Economics and Statistics, University of Salerno and CSEF, Via Giovanni Paolo II, 84084 Fisciano (SA), Italy 
are. This phenomenon is known as optimism bias and is defined as the difference between a person's expectation and the outcome that follows. If expectations are better than reality, the bias is optimistic. But expectations could be also worse than reality, in which case there is a pessimistic bias. The available evidence shows a prevalence of the optimistic bias, which might be problematic for both behavior and health. Dillard et al. (2009) show, for example, that overoptimism about avoiding alcohol-related problems corresponds with greater alcohol consumption. Radcliffe and Klein (2002), in studying the individual perception of a heart-attack risk, find that $56 \%$ of the people involved in the study are unrealistically optimistic, $25 \%$ unrealistically pessimistic, and only $19 \%$ accurate. Similarly, Krosnick et al. (2017), in a study investigating people's perception of getting lung cancer if they smoke, show that $54.6 \%$ of the respondents vastly underestimate it, while $23.9 \%$ overestimate it. $^{1}$

In this paper, we study the effects of an optimism bias on the consumption of sin goods, i.e., goods whose consumption generates negative health consequences in the future (O'Donoghue \& Rabin 2006), and the effectiveness of taxation and education in mitigating them. The importance of a multicomponent strategy in affecting sin goods consumption has been a topical issue not only in the academic but also in public debate (Le Bodo et al., 2016).

To this aim, we construct a model in which a risk averse agent has an optimism bias that causes a misperception of the health damages of sin goods consumption. This behavioral bias causes an underestimation of the health consequences in optimistic consumers and an overestimation in pessimistic ones. Within this setting, we study the way in which a social planner can affect the demand of such goods through taxation and education initiatives. The latter are referring to educational campaigns and disclosure requirements (warning labels on tobacco, alcohol or fatty food), and have the effect of increasing the consumers' awareness and align their perception of the health damages related to the consumption of such goods to the actual one. ${ }^{2}$

Although the literature has mainly focused on the effects of overoptimism (Roll, 1986; Malmendier \& Tate, 2005, 2008; Bénabou, 2012), less attention has been paid to the impact of the minority of pessimistic agents. Thus, after analyzing the benchmark case in which there are only optimistic consumers, and after determining the optimal mix of education initiatives and taxation needed to correct the consumers' behavioral bias, we introduce fully rational and pessimistic consumers and show that a fraction, however small, of them may reduce the effectiveness of such policies. In particular, the correcting effect that taxation has on optimistic consumers has the side effect of depressing the consumption of the fully rational and the pessimistic ones. As argued by Allcott et al. (2019a), a consumer might rationally consume the sin good despite the health risks, because enjoyment of the good outweighs the health harms. But the presence of both rational and pessimistic consumers in a setting in which they are mainly optimistic highlights one possible flaw of taxation. Namely, while correcting the excess consumption of optimistic consumers, taxation

\footnotetext{
1 Other studies have revealed similar findings for breast cancer (Waters et al., 2011).

2 A description of education initiatives and of the literature testing their effectiveness is provided in the next section.
} 
may worsen the underconsumption problem of pessimistic ones and distort the otherwise first-best consumption of rational ones. Education, instead, by aligning the biased consumers' perception of the health damages to the actual one, not only corrects both the optimistic and pessimistic consumers' behavioral bias, while leaving unaltered the choices of the rational ones, but also mitigates the distorting effects of taxation.

In modeling taxation and education measures, we assume that they are both costly. In particular, the cost of taxation is related to the inefficiency of the fiscal system translating one euro of tax revenues into less than one euro transferred to consumers due to administrative and compliance cost. Education initiatives, instead, have material costs and may generate a disutility on consumers for managing and processing the information received. ${ }^{3}$

Focusing on a simple quasi-linear economy in which there is one composite and one sin good, we find that if the fiscal system is not too inefficient and consumers are sensitive to information provision, taxation and education are both relied upon. Moreover, as the degree of inefficiency of the fiscal system varies, the two instruments can be substitutes or complements, depending on the impact of such variation on the deadweight loss of taxation and on the relative cost of education. While this latter effect is always negative on taxation and positive on education, the impact of an increased inefficiency of the fiscal system on the deadweight loss of taxation is ambiguous and depends on the elasticity of demand of the sin good with respect to taxation. It turns out that when the demand is inelastic, the marginal cost of taxation is positive and increasing in the degree of inefficiency of the fiscal system. For both the effects highlighted above, an increased inefficiency of the fiscal system makes room for education initiatives at the expense of taxation and the two instruments are substitutes. This result is in line with what one would expect following an increased inefficiency of the fiscal system, but it may be overturned when the demand is elastic. In this case the marginal cost of taxation becomes negative and decreasing in the degree of inefficiency of the fiscal system. For a sufficiently elastic demand, this makes room for an increased reliance on taxation. As regards education, this is not crowded out yet by the higher efficiency of taxation and, for a range of values of the elasticity, the two instruments are both effective in reducing the deadweight loss of taxation, becoming complements. Finally, when the demand is very elastic, a higher inefficiency of the fiscal system causes a reduction in the deadweight loss of taxation so high as to offset the positive effect that the higher relative cost of taxation has on education, determining a decreased reliance on education and an increased reliance on taxation.

We then introduce heterogeneous consumers and show another novel result, namely that in the presence of both rational and pessimistic agents the correcting effect of taxation in mitigating overconsumption of optimistic agents has unintended depressing effects on the choice of all the others. This asymmetry is not at work when education is used because of its effectiveness in aligning both optimistic and

\footnotetext{
${ }^{3}$ In the conclusions we discuss the possibility that education initiatives not only involve a disutility on consumers, but may be also costly for the society.
} 
pessimistic consumers' perception to the true probability of health damages, while leaving unaltered the choice of the rational ones.

The paper is organized as follows. After concluding this section with a brief description of literature and education initiatives, in Sect. 2, we set up the model focusing on optimistic consumers. In Sect. 3, we study the optimal mix of education initiatives and taxation. In Sect. 4, we analyze how this mix is affected by the presence of heterogeneous consumers. In the last section, we conclude. All the proofs are in Appendix.

\subsection{Related literature}

The paper is related to different strands of the literature. The first is that on time inconsistency and hyperbolic discounting (Ainslie, 1992; Laibson, 1997) studying the welfare effects of sin good regulation (Frederick et al., 2002; O'Donoghue \& Rabin, 2003, 2006; Gruber \& Koszegi, 2001, 2004; Gruber \& Mullainathan, 2005; Immordino et al., 2019). In particular, Immordino et al. (2019) study the optimal taxation and regulation of sin goods consumption when individuals' time inconsistency determines a problem of overconsumption. In the present paper, government intervention, through both taxation and educational measures, is called to correct the distortions induced by an optimism bias that may determine a problem both of overconsumption and underconsumption. However, unlike taxation that distorts the consumption problem of rational individuals and exacerbates the underconsumption problem of pessimistic ones, education may be effective in correcting both underconsumption and overconsumption problems. By focusing on the role of education, the paper is close to Mariotti et al. (2019), who study the optimal design of information nudges for present-biased consumers. However, despite the common information provision denominator, the focus of the two contributions diverge. Indeed, while Mariotti et al. (2019) ask which groups to address with such campaigns, our aim is to compare the relative merits of taxation and education.

Another related strand of the literature is that on overconfidence. Bénabou and Tirole (2002) have pointed out the trade-off between the value placed by rational agents on self-confidence, through an enhanced motivation, and the cost of the same, through self-deception. Other papers have studied the effects that managers with behavioral biases have on corporate decision making. For instance, Goel and Thakor (2008) show that overconfident managers are more likely to be promoted to CEOs than perfectly rational ones because they perceive less risk and so take more chances. Moreover, a moderate degree of overconfidence is beneficial, mitigating the problem of underinvestment that plagues strictly rational managers. The benefits of overconfidence and moderate overoptimism are also highlighted by Gervais et al. (2003), who show that both these traits help offset the excessive prudence induced by risk aversion, inducing managers to take investment decisions less hesitantly. Unlike this literature, in the present paper overoptimism is always detrimental as agents underestimate the probability of health damages and are induced to consume too much of the unhealthy good. 
The paper is also related to the literature studying the effect of information provision on consumption choices. In particular the literature on environmental externalities has focused on information provision as a policy instrument to supplement environmental taxation. Petrakis et al. (2005), in particular, show that information provision could dominate, in some cases, environmental taxation in terms of welfare and that a combination of the two policies is welfare improving. In addition, within a dynamic framework in which consumers' behavior depends on the accumulated stock of information rather than on currently provided information, Sartzetakis et al. (2012) show that information provision tends to reduce the optimal tax rate that declines over time as the accumulated stock of information increases. However, unlike our paper, in these contributions consumers do not have a behavioral bias but hold imperfect information about the damages related to the consumption of a polluting good, i.e., a good that generates individual and external environmental damages.

The positive role of information provision has also been studied also by Allcott and Taubinsky (2015) within a setting in which consumers hold wrong beliefs. Using two randomized experiments in which consumers become better informed about energy efficiency policies, they work out the welfare effects of an optimal subsidy as opposed to a quantity restriction policy. However, their focus differs from ours as they do not compare the efficiency of information provision relative to other instruments.

In the following section we give a brief overview of some evidence on education initiatives.

\subsection{Evidence on education initiatives}

Everyday life provides us with several examples of initiatives aimed at forbidding, limiting or deterring the consumption of sin goods. These initiatives can be categorized into two groups: education initiatives, aimed at increasing the awareness of the health effects of the consumption of sin goods, such as information/awareness campaigns, and policy initiatives, aimed at directly limiting the availability and provision of sin goods, such as consumption restrictions.

Education initiatives are widely used. In many countries, for example, health warning labels appear on fatty food, tobacco or alcohol products pointing to the health risks associated with their consumption, and are often mandatory. On the packaging of cigarettes and other tobacco products a variety of textual and pictorial warnings appear, covering, within a black frame, a large part of the surface of the pack and concerning the health effects of tobacco products consumption. For fatty food, some countries (e.g., the UK) have developed a system of front of pack nutritional labels that associates colors with information on fat, salt, sugar, and calories contained in food products, to help people make healthier choices. In some cases education initiatives can take the form of recommendations, like in responsible drinking and gambling campaigns to prevent alcoholism and ludopathy.

Educational initiatives seem to be effective in increasing the consumers' knowledge and attitude about the health consequences of the consumption of sin goods. 
Some empirical studies have focused on the effectiveness of educational campaigns on sin good consumption, especially on smoking. Hsieh et al. (1996), for example, have found that anti-smoking campaigns have a significantly positive effect on the public's health knowledge, i.e., on the awareness of the harmful effects of cigarette smoking, and that this health knowledge in turn, has a significantly negative effect on smoking participation. However, such policies may not always be effective. As highlighted by Gruber (2001), youths appear to think that they will not be addicted to smoking. In such cases, rather than emphasizing the long-term costs of smoking, information campaigns should "highlight the short-run implications of smoking in terms of reduced physical performance, appearance, and other costs directly salient to youths.",

Some evidence on the effectiveness of information provision is also available for food products. Kana and Tsai (2004) show the existence of a relation between individuals' knowledge on obesity's health risks and their tendency to be overweight. Variyam and Cawley (2006) study the impact on body weight and obesity of the law passed in 1990 in the USA imposing to manufacturers of packaged foods the disclosure of information about calories and nutrients (Nutrition Labeling and Education Act). They find that the release of this information is associated with a decrease in body weight and obesity. Cioffi et al. (2015) show that the introduction of food labels on a sample of pre-packaged food items results in a reduction of the average calories purchased from the labelled foods. More recently, Moran and Roberto (2018) study the effect of warning labels on beverages and show that they significantly reduce consumption of fruit drinks due to changes in health beliefs and risk perceptions.

\section{The model}

We study a setting in which a representative optimistic risk averse agent has to choose the optimal level of consumption of a sin good, i.e., a good that is enjoyable to consume but may create negative health consequences, such as alcohol, cigarettes, potato chips. The agent's utility is quasi-linear with respect to the sin $\operatorname{good}(x)$ and a composite good which acts as a numeraire (z). Specifically,

$$
u(x, z)= \begin{cases}v(x)-c(x)+z & \text { with probability } q \\ v(x)+z & \text { with probability } 1-q\end{cases}
$$

The function $v(\cdot)$ represents the benefit from sin good consumption and satisfies Inada conditions. The function $c(\cdot)$ represents the uncertain negative health

\footnotetext{
${ }^{4}$ For comprehensive review studies on the effectiveness of strategies and interventions aimed at preventing smoking uptake or alcohol-related problems see, for instance, Thomas et al. (2013) and Babor et al. (2003).
} 
consequences from sin good consumption and is such that $c_{x}>0, c_{x x}>0$, and $c_{x}(x)=0$ when $x=0 . .^{5}$ The parameter $q \in(0,1]$ is the (true) probability of getting into a certain state of health, henceforth referred to as probability of health damages. To preserve the empirically desirable feature of decreasing absolute risk aversion for any $q$, we also assume that $v_{x x x} \geq 0$ and $c_{x x x} \leq 0 .^{6}$

Agents feature an optimistic bias in that they underestimate the probability of health damages. We denote by $q^{o}<q$ the agent's perceived probability that the sin good causes health damages. ${ }^{7}$ Thus, the agent maximizes the optimistic expected utility function:

$$
U^{o} \equiv v(x)-q^{o} c(x)+z,
$$

subject to the budget constraint $I=p_{x} x+p_{z} z$, where $I$ is the exogenous income earned by the consumer, $p_{x}$ and $p_{z}$ are the prices of the sin good and of the numeraire, respectively. We assume that there is no borrowing or lending, that markets are competitive and that the marginal cost of producing both goods is equal to one, so that the price of each good is also one. ${ }^{8}$

Because of overoptimism, the consumer does not maximize his own expected welfare, measured by the actual expected utility function

$$
U=v(x)-q c(x)+z .
$$

In the following, we will call optimistic utility the expected utility function corresponding to the choice of the agent $\left(U^{o}\right)$, and actual utility the expected utility function that correctly reflects the expected welfare of the agent $(U)$.

The first-best consumption, which we denote by $\left(x^{F B}, z^{F B}\right)$, maximizes the actual utility $U$, subject to the budget constraint $I=x+z$, and is such that $x^{F B}$ satisfies the first-order condition $v_{x}\left(x^{F B}\right)-q c_{x}\left(x^{F B}\right)=1$ and $z^{F B}=I-x^{F B}$.

In the absence of policy measures aimed at affecting the consumers' behavior, the agent's sin good consumption, $x^{0}$, satisfies the first-order condition $v_{x}\left(x^{o}\right)-q^{o} c_{x}\left(x^{o}\right)=1$. Since $v_{x}$ is decreasing in $x, c_{x}$ is increasing in $x$ and $v_{x}(x)-q c_{x}(x)$ is lower than $v_{x}(x)-q^{o} c_{x}(x)$ for any $x, x^{o}$ is larger than $x^{F B}$. Moreover, $z^{o}=I-x^{0}$ is lower than $z^{F B}$. Thus, because of overoptimism, the agent consumes too much of the sin good and too little of the numeraire.

In the next section, we focus on the realistic case in which education initiatives can be combined with a linear tax on the consumption of the sin good to mitigate the consequences of the consumers' optimistic bias.

\footnotetext{
5 These assumptions guarantee that the consumer's problem is well behaved and are made to simplify the exposition. In particular, the assumptions that $\lim _{x \rightarrow 0} v_{x}(x)=\infty, \lim _{x \rightarrow \infty} v_{x}(x)=0$, and $c_{x}(x)=0$ when $x=0$ ensure that the sin good demand is strictly positive for any price $p_{x}<\infty$.

${ }^{6}$ For the empirical relevance of this assumption, see Guiso and Paiella (2008).

7 We are ignoring the possibility that consumption may display addictive behavior, in particular, the consumer's current utility might depend on a measure of past consumption (Becker \& Murphy, 1988; Gruber $\&$ Koszegi, 2004). We will discuss the effect of relaxing this assumption in the conclusions.

8 We assume that $I$ is large relative to the sin good consumption, so as to avoid corner solutions for $x$.
} 


\section{Education initiatives and taxes}

Education initiatives are all the regulatory measures aimed at creating the conditions for consumers to voluntarily limit consumption. To model them, we assume that the government can adopt an informational campaign aimed at increasing the consumers' awareness of the expected negative health consequences of sin good consumption and thereby affect the same. Such campaigns may be more or less effective, depending on the sensitivity of individuals to information, due, for example, to limited attention, and generate a disutility on consumers in the form of a cost in absorbing and processing information. ${ }^{9}$

The level of information provision is captured by the parameter $\gamma \in[0, \bar{\gamma}]$, where $\gamma=0$ means the absence of educational measures and $\gamma=\bar{\gamma}$ implies full awareness of the health damages of sin good consumption, i.e., $q^{o}=q$, provided that the consumers are fully sensitive to education initiatives. The sensitivity to the new information provision is measured by the parameter $\alpha \in[0,1]$, with $\alpha=0$ meaning that consumers are not sensitive to any informational program. Formally:

Assumption 1 The benefit of information provision is given by a continuous function $q^{o}(\gamma, \alpha) \equiv q^{o}+\alpha \delta(\gamma)$ defined on $[0, \bar{\gamma}] \times[0,1]$, with $\delta(\gamma)$ strictly increasing and concave, and equal to 0 when $\gamma=0$ and to $q-q^{o}$ when $\gamma=\bar{\gamma}$. The disutility of education is given by a continuous function $b(\gamma)$ defined on $[0, \bar{\gamma}]$, with $b(\gamma)$ strictly increasing and convex, and such that $b(0)=b_{\gamma}(0)=0$.

The parameter $\alpha$ is intended to capture the individual characteristics regarding the sensitiveness to information, which are exogenous and do not depend on $\gamma$. For example, individuals that are very sensitive to the issues concerning the consequences on their health of consuming junk food are more attentive to the information received on the matter and display a high value of $\alpha$.

The disutility of information $b(\gamma)$ captures the cost of processing and absorbing information (e.g., the time and effort spent in deciphering the calories intakes reported on food labels). ${ }^{10}$ It is interesting to notice that $\alpha$ and $b(\gamma)$ play a different role in the model. In particular, $\alpha$ enters directly the marginal utility of sin good consumption, affecting its level. Conversely, $b(\gamma)$ affects the optimal level of information provision $\gamma$, and thus only indirectly the level of consumption.

As for the health cost of sin good consumption, we assume that education has a disutility only for the individual and not for the society. ${ }^{11}$

The sin tax in our model is a linear tax $\tau$ on the consumption of the sin good. The effect of taxation is to limit the consumption of the sin good by increasing its price,

\footnotetext{
9 For a discussion on the issue of limited attention in consumer preferences, see Della Vigna (2009).

${ }^{10}$ In many real-life examples individual characteristics, $\alpha$, and disutility of information, $b(\gamma)$, might be correlated. However, since the model would quickly become untractable, we have chosen to model them as independent.

11 We will discuss the effect of relaxing these assumptions in the concluding section.
} 
which becomes $p_{x}=1+\tau$. We assume that the tax proceeds $\tau x$ are redistributed in a lump sum way to consumers, but there are inefficiencies associated with taxation in terms of administrative and compliance costs. ${ }^{12}$ This is formalized by assuming that one euro in tax revenues translates into less than one euro in transfers for consumers:

Assumption 2 The per-capita transfer $l$ from tax proceeds is given by:

$$
l=(1-\lambda) \tau x
$$

where $\lambda \in[0,1 / 2)$ is the direct inefficiency of the tax system, reflecting the loss in the economy from collecting one euro in tax revenues.

This assumption, along with that of decreasing absolute risk aversion, guarantees the concavity of the optimization problem. However, our qualitative results also hold in a more general setting.

When educational measures $\gamma$, linear tax $\tau$, and lump sum transfer $l$ are introduced, the agent's optimal consumption level maximizes his optimistic utility $U^{o}(\gamma)=v(x)-q^{o}(\gamma) c(x)-b(\gamma)+z$, subject to the budget constraint $z=I+l-(1+\tau) x$. Then, the optimal sin good consumption satisfies the first-order condition

$$
v_{x}(x)-q^{o}(\gamma, \alpha) c_{x}(x)=(1+\tau) .
$$

Let $x^{o}(\gamma, \tau)$ be the agent's consumption rule of the sin good defined by condition (3) and $z^{o}(\gamma, \tau)$ be the consumption rule of the composite good defined by the budget constraint. Since education improves the optimistic consumer awareness $q^{o}(\gamma, \alpha)$ when he is sensitive to the informational program $(\alpha>0)$, and taxation increases the price of the sin good, the agent's consumption rule $x^{o}(\gamma, \tau)$ is lower than $x^{o}$ for any policy pair $(\gamma, \tau)$ involving strictly positive education and/or taxation. Indeed, the first derivative of $x^{o}(\gamma, \tau)$ with respect to $\gamma$ is $x_{\gamma}^{o} \equiv \frac{\alpha \delta_{\gamma}(\gamma) c_{x}\left(x^{o}(\gamma, \tau)\right)}{v_{x x}\left(x^{o}(\gamma, \tau)\right)-q^{o}(\gamma, \alpha) c_{x x}\left(x^{o}(\gamma, \tau)\right)}<0$ for all $\alpha>0$, and the first derivative with respect to $\tau$ is $x_{\tau}^{o} \equiv \frac{1}{v_{x x}\left(x^{o}(\gamma, \tau)\right)-q^{o}(\gamma, \alpha) c_{x x}\left(x^{o}(\gamma, \tau)\right)}<0$.

The social planner chooses the level of education, $\gamma$, and the level of taxation, $\tau$, that maximize the actual expected utility function $U(\gamma)=v(x)-q c(x)-b(\gamma)+z$, subject to the budget constraints and to the lump-sum transfer constraint defined by condition (2), and the consumption rule defined by condition (3). ${ }^{13}$

By substituting the budget constraint, the lump-sum transfer constraint, and the consumption rule in the objective function, the latter reads as

\footnotetext{
12 The first, incurred by the tax authority to collect taxes and enforce fiscal laws, has been estimated to be $0.5 \%$ of net revenue collection for the USA, with a median of about $1 \%$ for OECD countries (OECD, 2011). For compliance costs, a study by PricewaterhouseCoopers (2015) for 189 countries across the world reports an average of $99 \mathrm{~h}$ spent by consumers to comply with sales tax and VAT.

13 An alternative way to analyze this problem could be the optimal Ramsey taxation approach (see, for instance, Chari \& Kehoe, 1999).
} 


$$
\Omega(\gamma, \tau)=\underbrace{\left[v\left(x^{o}(\gamma, \tau)\right)-q c\left(x^{o}(\gamma, \tau)\right)+I-x^{o}(\gamma, \tau)\right]}_{B(\gamma, \tau)}-\underbrace{\lambda \tau x^{o}(\gamma, \tau)}_{C T(\gamma, \tau)}-\underbrace{b(\gamma)}_{C E(\gamma)}
$$

For any policy pair $(\gamma, \tau)$, the term in square brackets represents the benefit of consumption, $B(\gamma, \tau)$. The second term, $C T(\gamma, \tau)$, represents the cost of taxation in terms of reduced consumption of the numeraire due to the inefficiency of taxation, while the third term, $C E(\gamma)$, represents the cost of education.

Let us define with $\left(\gamma^{*}, \tau^{*}\right)$ the policy pair maximizing objective function (4) and with $\hat{\gamma}=\arg \max \hat{\Omega}(\gamma)$, where $\hat{\Omega}(\gamma) \equiv \Omega(\gamma, \tau=0)$, the optimal level of education in the case where only education initiatives can be used by the social planner to mitigate the consequences of the consumers' optimistic bias. ${ }^{14}$ Then, our first result follows:

Proposition 1 When $\alpha>0$, (i) the optimal level of information provision is zero if $\lambda=0$ and is strictly positive if $\lambda>0$, (ii) there exists a threshold $\bar{\lambda}>0$ such that if $\lambda \in(0, \bar{\lambda})$, the optimal policy involves both education and taxation, i.e., $\gamma^{*}>0$ and $\tau^{*}>0$, (iii) $\gamma^{*}$ is lower than $\hat{\gamma}$ and $\tau^{*}$ is lower than $\hat{\tau}$. When $\alpha=0$, the optimal level of information provision is always zero and there exists a threshold $\lambda^{M} \geq \bar{\lambda}$ such that if $\lambda<\lambda^{M}$, the optimal policy involves strictly positive taxation, $\hat{\tau}$.

Proposition 1 states that as long as optimistic consumers are sensitive to educational initiatives and taxation is costly, information provision is always Pareto improving and, if the fiscal system is not too inefficient $(\lambda<\bar{\lambda})$, the regulator prefers to use both instruments. Moreover, relative to the case in which only education can be used, the possibility to use also taxation allows the social planner to reduce the reliance on education, thereby saving on education cost $\left(\gamma^{*}<\hat{\gamma}\right)$. However, if consumers are not sensitive to informational programs $(\alpha=0)$, then the social planner chooses not to provide any information and to use only sin taxes, provided the deadweight loss of taxation is sufficiently low. ${ }^{15}$

Proposition 1 predicts that for small values of $\alpha$ information campaigns still tend to be strictly positive (if $\lambda>0$ ). This result relies on the assumptions that $b_{\gamma}(0)=0$ and $b(0)=0$. However, if $b_{\gamma}(0)>0$ and/or $b(0)>0$, we expect that the social planner does not take any educational measure when consumers are not very sensitive to informational programs ( $\alpha$ small), since the marginal cost of education and/or its fixed component may offset the benefits of information campaigns. This points out that the sensitivity to new information, $\alpha$, and the disutility of education, $b(\gamma)$, are strictly related and underlines the crucial role that the latter plays in the choice of the optimal policy instruments. ${ }^{16}$

\footnotetext{
14 For a formal derivation of $\hat{\gamma}$ see Lemma 1 in Appendix.

15 The results for the case in which $\alpha=0$ are equivalent to those obtained by Immordino et al. (2019) in which the overconsumption problem is due to agents' hyperbolic preferences.

16 To gain an intuition, consider the case where the disutility of education is given by the affine function $b(\gamma)=b_{0}+b_{1} \gamma$, with $b_{0} \geq 0$ and $b_{1}>0$. Under this hypothesis, there is a sensitivity threshold, $\underline{\alpha} \leq 1$, such that if $\alpha$ is below $\alpha$ it is not optimal to provide information since the marginal benefit of education, that is, the increase in the consumer's expected welfare due to the reduced sin good consumption, is
} 
Since in our model the qualitative results are the same for all $\alpha>0$, for simplicity we restrict the analysis to the case in which $\alpha=1$. Proposition 1 sets a critical value for the inefficiency of the fiscal system below which the instruments are both relied upon $(\lambda<\bar{\lambda})$. However, the way in which inside this range of values the reliance on both instruments varies as $\lambda$ varies depends on the elasticity of demand of the sin good with respect to taxation, $\eta_{x, \tau}=\frac{x_{\tau}^{o}(\tau, \gamma) \tau}{x^{o}(\tau, \gamma)}$, as stated in Proposition 2:

Proposition 2 Assume $\alpha=1$ and $\lambda \in(0, \bar{\lambda})$. There exist two thresholds $\bar{\eta}$ and $\eta$, with $\eta<\bar{\eta}<-1$, such that: (i) $\partial \tau^{*} / \partial \lambda \leq 0$ if and only if $\eta_{x, \tau} \geq \bar{\eta}$, and (ii) $\partial \gamma^{*} / \partial \bar{\lambda} \leq 0$ if $\overline{\text { and }}$ only if $\eta_{x, \tau} \leq \underline{\eta}$.

The intuition behind the above results can be understood by decomposing the total effect of a change in $\lambda$ on the two instruments in two effects. A first effect, capturing how a change in $\lambda$ affects the relative cost of education, and a second effect, capturing how a change in $\lambda$ affects the marginal deadweight loss of taxation, $\lambda \Delta(\tau x)$. The first effect is always negative on taxation and positive on education. This is because a change in $\lambda$, by increasing the cost of taxation, decreases the relative cost of education, thus making one instrument comparatively less expensive than the other. We call this the relative cost effect. As regards the second effect, which we call the demand elasticity effect, the way in which the marginal deadweight loss of taxation varies as $\lambda$ varies has an ambiguous sign as it depends on the elasticity of demand. When the demand is inelastic $\left(\eta_{x, \tau}>-1\right)$, the sin good consumption varies less than proportionally with $\tau$, which implies that both the proceeds from taxation and the deadweight loss of taxation increase in $\tau$. In this context, an increase in $\lambda$ further increases the deadweight loss of taxation, determining a lower reliance on taxation and a higher reliance on education. Thus, when the demand is inelastic, the demand elasticity effect has the same sign on each of the two instruments as the relative cost effect, which implies that the two effects reinforce each other (with education increasing and taxation decreasing in $\lambda$ ) and the instruments are substitutes.

If the demand is elastic $\left(\eta_{x, \tau} \leq-1\right)$, the sin good consumption varies more than proportionally with $\tau$, implying that both the proceeds from taxation and the deadweight loss of taxation decrease with $\tau$. In this context, an increase in $\lambda$ decreases the marginal deadweight loss of taxation, $\lambda \Delta(\tau x)$, and should result in an advantage of taxation over education, with a greater reliance on taxation and a lower reliance on education. However, for a value of elasticity close to -1 , this effect is small when compared with the relative cost effect (that makes education increasing and taxation decreasing in $\lambda$ ) and the two instruments are still substitutes. As the demand becomes more elastic, the decrease in the marginal deadweight loss of taxation

Footnote 16 (continued)

lower than the (constant) marginal disutility of education, $b_{1}$, for all $\gamma$. In addition, even for values of $\alpha$ greater than $\underline{\alpha}$ but sufficiently close to it, the social planner may still prefer to not provide any information due to its fixed cost, $b_{0}$. Finally, the higher the marginal disutility from regulation, the higher the sensitivity threshold making education measures Pareto improving. In particular, if $b_{1}$ is very high, then $\underline{\alpha}=1$ and it is never optimal to use information provision. 
implied by the increase in $\lambda$ is sufficiently high to offset the relative cost effect and make taxation increasing in $\lambda$. We denote the threshold value of $\eta_{x, \tau}$ below which this occurs as $\bar{\eta}$. As regards education, such offsetting demand elasticity effect is of a lower magnitude, implying that for $\eta_{x, \tau}=\bar{\eta}$, the reliance on education is still increasing in $\lambda$ due to the relative cost effect. Thus, for $\eta_{x, \tau}<\bar{\eta}$, the two instruments are complements. However, as the demand becomes even more elastic, the decrease in the marginal deadweight loss of taxation implied by the increase in $\lambda$ is so high as to offset also for education the relative cost effect and make education decreasing in $\lambda$. We denote the threshold value of $\eta_{x, \tau}$ below which this occurs as $\eta$. For such values, the two instruments are again substitutes. However, their use varies in a direction which is opposite to the expected one as an increase in $\lambda$ calls for an increased reliance on taxation and a lowered reliance on education.

From the above, we deduce that the relation between the two instruments depends on the elasticity of demand, as described in Corollary 1.

Corollary 1 The two instruments are complements for all $\eta_{x, \tau} \in(\eta, \bar{\eta})$, while they are substitutes otherwise. In particular, for $\eta_{x, \tau} \leq \underline{\eta}, \partial \tau^{*} / \partial \lambda \geq 0$ and $\partial \gamma^{*} / \partial \lambda \leq 0$, while for $\eta_{x, \tau} \geq \bar{\eta}, \partial \tau^{*} / \partial \lambda \leq 0$ and $\partial \gamma^{*} / \partial \lambda \geq 0$.

The above corollary shows that the way in which the reliance on both instruments varies as $\lambda$ varies depends on the elasticity of demand. When the demand is inelastic or not too elastic $\left(\eta_{x, \tau} \geq \bar{\eta}\right)$, the two instruments are substitutes and, for the mechanisms described above, education increases in $\lambda$ and taxation decreases in $\lambda$. When the demand is mildly elastic $\left(\eta_{x, \tau} \in(\eta, \bar{\eta})\right)$, an increase in $\lambda$ causes a decrease in the deadweight loss of taxation sufficiently high to result in a higher reliance on taxation, but education is not crowded out by the increased efficiency of taxation. Thus, the two instruments are both effective in reducing the deadweight loss of taxation and they become complements. When the demand is highly elastic $\left(\eta_{x, \tau} \leq \eta\right)$, the reduction in the deadweight loss of taxation is so high as to cause a decreased reliance on education. The two instruments are substitutes again, but with taxation increasing in $\lambda$ and education decreasing in $\lambda$.

\section{Heterogeneous consumers}

So far we have assumed that all consumers underestimate the likelihood of health damages caused by sin good consumption. In this section, we introduce heterogeneity across agents, namely, within a setting in which a fraction $\pi$ of consumers is rational and agents who feature a bias are mainly optimistic, we assume that a fraction $\mu$ of biased consumers is pessimistic and overestimates the probability of health damages. $^{17}$

\footnotetext{
17 Notice that the presence of rational and pessimistic agents implies that unlike the case in which there are only optimistic agents (see footnote 15), when education is ineffective $(\alpha=0)$, the results are not equivalent to those that would be obtained with hyperbolic preferences.
} 
We denote by $q^{p}>q$ the perceived probability that the sin good causes health damages for a pessimistic agent and, since we ignore the extent of the misperception suffered by optimistic and pessimistic consumers, we take a conservative stance and assume that it is symmetric, i.e., $\left|q^{p}-q\right|=\left|q^{o}-q\right| \equiv \bar{\delta}$, with $\bar{\delta}<\min \{q, 1-q\}$.

A rational agent maximizes the actual expected utility (1), subject to the budget constraint $I=x+z$. Indeed, the expected utility function which guides the choice of the unbiased agent is the actual utility which correctly reflects the expected welfare of all consumers. Hence, in the absence of policy measures aimed at affecting the consumers' behavior, an unbiased agent consumes the first-best quantities $\left(x^{F^{B}}, z^{F B}\right)$.

An agent who overestimates the probability of health damages maximizes the pessimistic expected utility:

$$
U^{p} \equiv v(x)-q^{p} c(x)+z
$$

subject to the budget constraint. Hence, his sin good consumption, $x^{p}$, satisfies the first-order condition $v_{x}\left(x^{p}\right)-q^{p} c_{x}\left(x^{p}\right)=1$. Since $v_{x}$ is decreasing in $x, c_{x}$ is increasing in $x$ and $v_{x}(x)-q c_{x}(x)$ is greater than $v_{x}(x)-q^{p} c_{x}(x)$ for any $x, x^{p}$ is lower than $x^{F B}$. Moreover, $z^{p}=I-x^{p}$ is larger than $z^{F B}$. Thus, because of pessimism, the agent consumes too little of the sin good and too much of the numeraire.

In what follows, we consider two settings: one in which only educational policies are available to correct consumers' misbehavior, and a second one in which both education and taxation can be used.

Only education initiatives Education provides information useful to improve the consumers' awareness and align the consumers' perception of the probability of health damages to the actual one. Formally, the parameter $\gamma$ raises the awareness of the "true" $q$ both for optimistic and pessimistic consumers.

Assumption 3 The benefit of information provision is given by a continuous function

$$
q^{a}(\gamma) \equiv\left\{\begin{array}{l}
q^{o}+\delta(\gamma) \quad \text { if the agent is optimistic }(a=o) \\
q^{p}-\delta(\gamma) \text { if the agent is pessimistic }(a=p)
\end{array}\right.
$$

with $\delta(\cdot)$ defined on $[0, \bar{\gamma}]$, strictly increasing and concave, and equal to 0 when $\gamma=0$ and to $\bar{\delta}$ when $\gamma=\bar{\gamma}$. The disutility of regulation is given by a continuous function $b(\gamma)$ defined on $(0, \bar{\gamma}]$ with $b(\gamma)$ strictly increasing and convex, and such that $\lim _{\gamma \rightarrow 0} b(\gamma)=0$.

Under Assumption 3, when educational policies are adopted, the agent's unbiased utility becomes

$$
U(\gamma)=v(x)-q c(x)-b(\gamma)+z,
$$

the agent's optimistic utility becomes

$$
U^{o}(\gamma)=v(x)-(q-\bar{\delta}+\delta(\gamma)) c(x)-b(\gamma)+z,
$$


and the agent's pessimistic utility becomes

$$
U^{p}(\gamma)=v(x)-(q+\bar{\delta}-\delta(\gamma)) c(x)-b(\gamma)+z .
$$

An increase in $\gamma$ has zero effect on the marginal unbiased utility, a negative effect on the marginal optimistic utility and a positive effect on the marginal pessimistic utility $\left(U_{x \gamma}^{o}(\gamma)=-\delta_{\gamma} c_{x}<U_{x \gamma}(\gamma)=0<U_{x \gamma}^{p}(\gamma)=\delta_{\gamma} c_{x}\right)$.

In the case of information provision $\gamma$, the optimal consumption bundle of a type $a$ biased agent, which we denote by $\left(x^{a}(\gamma), z^{a}(\gamma)\right)$, with $a \in\{o, p\}$, maximizes $U^{a}$ subject to $z=I-x$. Then, the consumption rule of the sin good, $x^{a}(\gamma)$, satisfies the first-order condition

$$
v_{x}(x)-q^{a}(\gamma) c_{x}(x)=1 .
$$

Moreover, the optimal consumption bundle of an unbiased agent is $\left(x^{u}(\gamma), z^{u}(\gamma)\right)=\left(x^{F B}, z^{F B}\right)$, since regulation does not affect his marginal utility.

The social planner chooses the level of information provision $\hat{\gamma}^{h}$ that maximizes the expected welfare

$$
\begin{aligned}
U^{h}(\gamma)= & \pi\left(v\left(x^{F B}\right)-q c\left(x^{F B}\right)+z^{F B}\right)+ \\
& +(1-\pi)\left[\mu\left(v\left(x^{p}(\gamma)\right)-(q+\bar{\delta}-\delta(\gamma)) c\left(x^{p}(\gamma)\right)+z^{p}(\gamma)\right)+\right. \\
& \left.+(1-\mu)\left(v\left(x^{o}(\gamma)\right)-(q-\bar{\delta}+\delta(\gamma)) c\left(x^{o}(\gamma)\right)+z^{o}(\gamma)\right)\right]-b(\gamma)
\end{aligned}
$$

subject to the budget constraints $I-x^{F B}=z^{F B}, \quad I-x^{o}(\gamma)=z^{o}(\gamma), \quad$ and $I-x^{p}(\gamma)=z^{p}(\gamma)$. $^{18}$

By substituting the budget constraints in the expected welfare, the latter can be written as

$$
\hat{\Omega}^{h}(\gamma)=\underbrace{\left[\pi B I^{u}+(1-\pi)\left(\mu B I^{p}(\gamma)+(1-\mu) B I^{o}(\gamma)\right)\right]}_{B I^{h}(\gamma)}-C E^{h}(\gamma),
$$

where $\quad B I^{u} \equiv v\left(x^{F B}\right)-q c\left(x^{F B}\right)-x^{F B}+I, \quad B I^{p}(\gamma) \equiv\left(v\left(x^{p}(\gamma)\right)-q c\left(x^{p}(\gamma)\right)-x^{p}(\gamma)+I\right)$, $B I^{o}(\gamma) \equiv\left(v\left(x^{o}(\gamma)\right)-q c\left(x^{o}(\gamma)\right)-x^{o}(\gamma)+I\right)$, and $C E^{h}(\gamma) \equiv b(\gamma)$.

The term in square brackets, $B I^{h}(\gamma)$, is the expected benefit of consumption. The second term, $C E^{h}(\gamma)$, represents the cost induced by the disutility of information provision.

The social planner's problem is to choose $\hat{\gamma}^{h}$ that maximizes the distance between the benefits and the costs associated with information provision. To make the problem tractable and ensure concavity, we restrict the analysis to the case where only a small fraction of agents is pessimistic and assume $c_{x x}<\varepsilon$, with $\varepsilon$ positive and close to 0 .

\footnotetext{
${ }^{18}$ We use a social welfare function that puts equal weight on all agents, that is, the expectation of the individual experienced utility.
} 
Proposition 3 Suppose there is a fraction $\pi$ of unbiased consumers in the population and assume that a small fraction $\mu>0$ of biased consumers is pessimistic. If only education is available, the optimal level of information provision is $\hat{\gamma}^{h}<\hat{\gamma}$, with $\frac{\partial \hat{\gamma}^{h}}{\partial \pi}<0$.

By reducing the distance between perceived and actual likelihood of health damages (caused by sin good consumption) education makes the consumption of both optimistic and pessimistic agents closer to the first-best and increases utility without affecting the consumption of rational agents. In equilibrium, the marginal cost of information provision equals the average marginal benefit, $B I_{\gamma}^{h} \equiv(1-\pi)\left(\mu B I_{x}^{p} x_{\gamma}^{p}+(1-\mu) B I_{x}^{o} x_{\gamma}^{o}\right)$, where $B I_{x}^{a} \equiv v_{x}(x)-q c_{x}(x)-1$ is the social planner's marginal utility evaluated at $x=x^{a}(\gamma)$ and $x_{\gamma}^{a} \equiv \frac{q_{\gamma}^{a} c_{x}\left(x^{a}(\gamma)\right)}{v_{x x}\left(x^{a}(\gamma)\right)-q^{a}(\gamma) c_{x x}\left(x^{a}(\gamma)\right)}$ is the effectiveness of information provision in aligning optimistic and pessimistic consumption to the first-best, with $a \in\{o, p\}$. Relative to the case where all consumers are optimistic, consumer heterogeneity modifies both i) the social planner's marginal utility and ii) the effectiveness of information provision. In particular, the costs of educational measures are borne by the entire population of consumers, while their benefits concern only the fraction $1-\pi$ of biased agents. Hence, the optimal level of information provision decreases as the fraction of unbiased agents increases. Moreover, as shown in the proof of Proposition 3, the social marginal benefit obtained by reducing the consumption of an optimistic agent, $\left|B I_{x}^{o}\right|$, is larger than the one obtained by increasing the consumption of a pessimistic agent, $\left|B I_{x}^{p}\right|$. As a consequence, the introduction of both unbiased and pessimistic consumers lowers the social planner's marginal utility. ${ }^{19}$ Similarly, the effectiveness of information provision decreases since educational measures do not affect the optimal consumption bundle of unbiased agents and are more effective in reducing the consumption of optimistic agents than in increasing that of pessimistic ones. As a result, the level of information provision is lower than that stated in Proposition 1, that is, $\hat{\gamma}^{h}<\hat{\gamma}$.

Education initiatives and taxes Consider now the case in which also taxation is available to correct consumers' misbehavior.

Notice that when consumers are heterogeneous, implementing the first-best outcome would require individual-specific taxes and subsidies even if the efficiency cost of taxation is absent, i.e., for $\lambda=0$. However, since individual-specific taxes and subsidies are unrealistic because of informational constraints and implementation costs, we limit our analysis to a uniform tax. Recall that the goal of taxation is to limit consumption (for optimistic individuals) by increasing the sin good price.

\footnotetext{
19 The proof relies on the distance $\left|x^{o}(\gamma)-x^{F B}\right|$ being larger than $\left|x^{p}(\gamma)-x^{F B}\right|$, so that from the concavity of the social planner utility function, the marginal benefit of reducing the sin good consumption of an optimistic agent, $\left|B I_{x}^{o}\right|$, is larger than the marginal benefit of increasing the sin good consumption of a pessimistic agent, $\left|B I_{x}^{p}\right|$.
} 
However, this has the undesired effect of reducing consumption also for rational and pessimistic agents. ${ }^{20}$

Let be $x^{a}(\gamma, \tau)$, with $a \in\{u, o, p\}$, the type $a$ agent's consumption rule of the sin good for any policy pair $(\gamma, \tau){ }^{21}$ The social planner chooses the level of education, $\gamma^{h}$, and the level of taxation, $\tau^{h}$, maximizing the distance between the expected benefits and costs, i.e.,

$$
B^{h}(\gamma, \tau)=\pi B I^{u}(\gamma, \tau)+(1-\pi)\left(\mu B^{p}(\gamma, \tau)+(1-\mu) B^{o}(\gamma, \tau)\right)
$$

and

$$
C T^{h}(\gamma, \tau)=\pi C^{u}(\gamma, \tau)+(1-\pi)\left(\mu C^{p}(\gamma, \tau)+(1-\mu) C^{o}(\gamma, \tau)\right),
$$

where $B I^{a}(\gamma, \tau) \equiv\left(v\left(x^{a}\right)-q c\left(x^{a}\right)-x^{a}+I\right), C^{a}(\gamma, \tau) \equiv \lambda \tau x^{a}+b(\gamma)$, and $x^{a} \equiv x^{a}(\gamma, \tau)$ for all $a \in\{u, o, p\}$.

To make the model tractable, we first characterize the optimal policy pair into a setting where there are only rational and optimistic consumers and then analyze how the presence of pessimistic consumers modifies the regulator's optimal choice.

Let us define with $\left(\gamma_{\mid \mu=0}^{h^{*}}, \tau_{\mid \mu=0}^{h^{*}}\right)$ the policy pair maximizing the distance between (6) and (7) under the assumption that there are no pessimistic consumers, i.e., $\mu=0$. The next proposition shows that information provision is always Pareto improving and, provided taxation is not too inefficient $\left(\lambda<\bar{\lambda}^{h}\right)$, the social planner chooses to use both instruments to mitigate the consequences of the consumers' bias.

Proposition 4 Assume $\mu=0$. If both education and taxation are available, the optimal level of information provision is strictly positive for all $\lambda \geq 0$ and there exists $a$ threshold $\bar{\lambda}^{h} \in(0, \bar{\lambda}]$ such that if $\lambda<\bar{\lambda}^{h}$, the optimal policy involves both education and taxation, i.e., $\gamma_{\mid \mu=0}^{h^{*}}>0$ and $\tau_{\mid \mu=0}^{h^{*}}>0$. Moreover, $\lim _{\pi \rightarrow 1} \bar{\lambda}^{h}=0$.

Proposition 4 points out that if a fraction of agents is unbiased, the regulator always uses educational policies, even under the assumption of zero administrative and compliance cost $(\lambda=0)$. The rationale for this result is that with consumers' heterogeneity, a uniform tax generates an endogenous cost due to the consumption distortion for rational agents. ${ }^{22}$ By reducing the reliance on taxation, educational initiatives lower such cost. Moreover, fiscal policy is rarely used when most consumers are rational. Indeed, the threshold level of administrative and compliance costs that makes the fiscal policy worthwhile, $\bar{\lambda}^{h}$, tends to zero if the fraction of optimistic agents is small $(\pi \rightarrow 1)$.

Proposition 5 states the other novel result of our paper, i.e., that the presence of a fraction, however small, of pessimistic agents lowers the effectiveness of taxation in mitigating overconsumption thereby inducing the social planner to boost up educational measures.

\footnotetext{
${ }^{20}$ Although in principle a large fraction of pessimistic agents in the sample could imply a negative tax (i.e., a subsidy), the proof of our main result assumes that the fraction of pessimistic agents is small, in line with the evidence, implicitly ruling out $\tau^{*}<0$.

21 For any $a \in\{u, o, p\}, x^{a}(\gamma, \tau)$ satisfies the first-order condition $v_{x}(x)-q^{a}(\gamma) c_{x}(x)=1+\tau$.

22 Such effect has also been highlighted in O'Donoghue and Rabin (2006).
} 
Proposition 5 Suppose there is a small fraction $\mu>0$ of pessimistic consumers in the population. If both education and taxation are available, the optimal level of taxation is $\tau^{h^{*}}<\tau_{\mid \mu=0}^{h^{*}}$ and the optimal level of information provision is $\gamma^{h^{*}}>\gamma_{\mid \mu=0}^{h^{*}}$.

The intuition behind this result can be grasped by considering that the optimal uniform tax for pessimistic agents would be negative. Thus, when both optimistic and pessimistic agents coexist the optimal tax level is smaller than the one prevailing when agents are all optimistic. The previous result also shows that the level of education is higher than the one that obtains with optimistic agents only, and the two instruments are substitutes. This can be ascribed to the fact that while education initiatives always get both optimistic and pessimistic consumers' perceptions closer to the true probability of health damages, taxation, although effective for optimistic types, distorts the choice of pessimistic ones. Moreover, relative to the case in which only education is available, information provision is more valuable when taxation is also available. This is because taxation increases the distance between the pessimistic and the first-best consumption level, thus increasing the social benefit of inducing pessimistic agents to consume more.

Finally, as already in Proposition 4, this result does not rest on the inefficiency of the tax system. Indeed, even in a fully efficient fiscal system $(\lambda=0)$, a tax levied on sin goods would still affect the consumption choice of pessimistic consumers in the "unwanted/undesired" direction, thus calling for the corrective effect of educational measures.

\section{Concluding remarks}

The paper studies the role of taxation and education in a setting in which an optimistic agent underestimates the probability of future health damages from consuming a sin good and thus consumes too much of the same. Depending on the elasticity of demand of the sin good with respect to taxation and the relative efficiency of education, the paper shows that these two instruments can be used as substitutes or complements. However, when agents are heterogeneous, namely there are optimistic consumers who underestimate the probability of health damages and consume too much, pessimistic ones, who overestimate it and consume too little, and fully rational ones, who consume the first-best quantity, the correcting effect that taxation has on optimistic consumers has unintended distorting effects on the choices of both rational and pessimistic ones. In this framework, educational measures, by aligning both optimistic and pessimistic consumers' perception closer to the true probability of health damages, are more effective than taxation and should be preferred. Thus, besides the relative efficiency of each instrument, the paper points to an additional and unexpected advantage of education in correcting the distorting effects of taxation.

Some comments are in order.

First, when talking about educational campaigns we refer to measures that a social planner may want to adopt to improve consumers' awareness. Of course the underlying idea is that these measures are fully effective in correcting consumers' 
misperception (be it positive or negative). In particular, the "selective" correcting effect of education is in line with the literature on nudges (Thaler \& Sunstein, 2003), positing the positive impact of indirect suggestions on consumers with behavioral biases, but its neutrality on fully rational ones. One should nevertheless not disregard the ongoing debate about the risks that hard and soft paternalistic policies, if erroneously calibrated, may distort choices, as highlighted, for example, by Glaeser (2006). This risk may exacerbate the underconsumption problem of pessimistic consumers and restore a role for taxes as a complementary policy tool.

Second, one could argue that paternalistic policies are rarely concerned with pessimistic consumers who are overly careful. Although this is true, our concern with pessimistic consumers is the natural consequence of our normative approach of maximizing the social welfare function rather than minimizing sin good consumption.

Last, there seems to be a link between biases in beliefs and time inconsistency as underconsumption or overconsumption of the sin good also results from consumers with present-biased or future-biased preferences. One could then ask whether education initiatives can effectively be used to correct the individuals' time inconsistency biases. Our conjecture is that the only effect of such initiatives would be to make the unsophisticated consumers aware of their bias, without altering their choices. To be effective, such initiatives should be paired with self-control techniques as a means of combating one's own time-inconsistent tendencies. ${ }^{23}$ In this respect, taxation has an advantage relative to education as it provides both the sophisticated and the unsophisticated present-biased consumers with the commitment device needed to modify their consumption choices.

The paper could be extended in various directions. First, we have assumed that both education and the health cost of sin good consumption are borne only by the individual and are not costly for the society. Actually, the implementation of education initiatives is costly and may involve an extra tax burden on consumers. As pointed out in Allcott et al. (2019b), this could be financed with sin tax revenues, thereby also compensating the regressive nature of such taxes. ${ }^{24}$

In addition, we have neglected that consumption may display addictive behavior, in particular, the consumer's current utility might depend on a measure of past consumption (Becker \& Murphy, 1988; Gruber \& Koszegi, 2004). Incorporating this feature into our model would require the setup of a dynamic model and would affect the relative effectiveness of each regulatory measure. In particular, since the marginal utility of consuming an addictive good increases with time, there is a limit to the effectiveness of education given by the knowledge of the true probability of health damages. Such drawback would not affect taxation, which can always be increased to offset the effect the decreased sensitivity of the consumer to the policy measures.

Last, throughout the paper, we have assumed that an optimism bias is always problematic, as it always distorts consumption. In fact this is not always the case, as

\footnotetext{
${ }^{23}$ Gruber \& Koszegi (2001) report several examples of voluntary use of self-control devices to quit smoking.

24 Examples include sports funding in schools (UK), children's health programs (Berkeley, USA), and kindergartens (Philadelphia, USA), all financed with the revenues from the soda tax.
} 
there are instances in which overoptimism may have beneficial effects on mental and physical health (Scheier \& Carver, 1985). This would reduce the scope for education measures aimed at correcting the optimistic bias.

These and other issues are left to future research.

\section{Appendix}

Lemma 1 When taxation is not used, the optimal level of information provision is $\hat{\gamma} \in(0, \bar{\gamma}]$, with $\hat{\Omega}_{\gamma}(\hat{\gamma})=0$ whenever $\hat{\gamma}<\bar{\gamma}$, if $\alpha>0$, and is $\hat{\gamma}=0$ if $\alpha=0$.

Proof of Lemma 1 Assume $\alpha>0$. When taxation is not used, the social planner's objective function (4) becomes $\hat{\Omega}(\gamma)=\left[v\left(x^{o}(\gamma)\right)-q c\left(x^{o}(\gamma)\right)+I-x^{o}(\gamma)\right]-b(\gamma)$, with the optimistic consumption rule of the sin good, $x^{o}(\gamma)$, satisfying the first-order condition $v_{x}(x)-q^{o}(\gamma, \alpha) c_{x}(x)=1$.

For all $\gamma \in[0, \bar{\gamma}]$, as long as $v$ and $c$ are thrice differentiable, $\hat{\Omega}(\gamma)$ is continuous and twice differentiable. If strictly positive and lower than $\bar{\gamma}, \hat{\gamma}$ satisfies the firstorder condition $\hat{\Omega}_{\gamma}(\hat{\gamma})=\hat{\Omega}_{x}^{o}(\hat{\gamma}) x_{\gamma}^{o}(\hat{\gamma})-b_{\gamma}(\hat{\gamma})=0$, where $\hat{\Omega}_{x}^{o}(\hat{\gamma})=\left[v_{x}\left(x^{o}(\hat{\gamma})\right)-q c_{x}\left(x^{o}(\hat{\gamma})\right)-1\right]$. From the first-order condition of the optimistic agent, we can derive $\hat{\Omega}_{x}^{o}=-\left(q-q^{o}(\gamma, \alpha)\right) c_{x}\left(x^{o}(\gamma)\right)<0$. When $\gamma=0, \quad \hat{\Omega}_{\gamma}=-\left(q-q^{o}\right) c_{x}\left(x^{o}\right) x_{\gamma}^{o}(0)>0$. Hence, $\gamma=0$ cannot be a corner solution of the social planner maximization problem and $\hat{\gamma} \in(0, \bar{\gamma}]$. Finally, $\hat{\Omega}_{\gamma \gamma}<0$. Indeed, $\hat{\Omega}_{\gamma \gamma}=\left[\hat{\Omega}_{x}^{o} x_{\gamma \gamma}^{o}+\hat{\Omega}_{x \gamma}^{o} x_{\gamma}^{o}\right]-b_{\gamma \gamma}=\left[\hat{\Omega}_{x}^{o} x_{\gamma \gamma}^{o}+\hat{\Omega}_{x x}^{o} x_{\gamma}^{o 2}\right]-b_{\gamma \gamma}$, with $\hat{\Omega}_{x x}^{o}=v_{x x}-q c_{x x}$ and $x_{\gamma y}^{o}=\frac{\left(q_{y \gamma}^{o} c_{x}+q_{\gamma}^{o} c_{x x} x_{\gamma}\right)\left(v_{x x}-q^{o}(\gamma, \alpha) c_{x x}\right)-\left(v_{x x}-q^{o}(\gamma, \alpha) c_{x x}\right) x_{\gamma}^{o} q_{\gamma}^{o} c_{x}+\left(q_{\gamma}^{o}\right)^{2} c_{x} c_{x x}}{\left(v_{x x}-q^{o}(\gamma, \alpha) c_{x x}\right)^{2}}$. Since by assumption $q_{\gamma}^{o}>0, c_{x}>0, v_{x x}-q^{o} c_{x x}<0, b_{\gamma \gamma} \geq 0, q_{\gamma \gamma}^{o} \leq 0$, and $v_{x x x}-q^{o} c_{x x x} \geq 0$, then $\hat{\Omega}_{x x}^{o}<0, x_{\gamma \gamma}^{o}>0$, and then $\hat{\Omega}_{\gamma \gamma}^{o}<0$. This proves the unicity of $\hat{\gamma}$.

Assume $\alpha=0$. When taxation is not used, the social planner's objective function (4) becomes $\hat{\Omega}(\gamma)=\left[v\left(x^{o}\right)-q c\left(x^{o}\right)+I-x^{o}\right]-b(\gamma)$, with the optimistic consumption rule of the sin good, $x^{o}$, satisfying the first-order condition $v_{x}(x)-q^{o} c_{x}(x)=1$, and $\hat{\Omega}_{\gamma}=-b_{\gamma}<0$ for all $\gamma$. Hence, $\hat{\gamma}=0$.

Lemma 2 Let $\hat{\Omega}(\tau) \equiv \Omega(\gamma=0, \tau)$. Assume that $q^{o}<q$ and $\lambda<\lambda^{M}$, with $\lambda^{M}=\min \left\{\frac{\left(q-q^{o}\right)\left|c_{\tau}\left(x^{o}\right)\right|}{x^{o}}, \frac{1}{2}\right\}$. When education is not used, the optimal level of taxation is $\hat{\tau}>0$ such that $\hat{\Omega}_{\tau}(\hat{\tau})=0$, where $\hat{\Omega}(\tau) \equiv \Omega(\gamma=0, \tau)$.

Proof of Lemma 2 For all $\tau \geq 0$, as long as $v$ and $c$ are thrice differentiable, $\hat{\Omega}(\tau)$ is continuous and twice differentiable. If strictly positive, $\hat{\tau}$ satisfies the first-order condition $\hat{\Omega}_{\tau}(\hat{\tau})=\hat{\Omega}_{x}(\hat{\tau}) x_{\tau}^{o}(\hat{\tau})-\lambda x^{o}(\hat{\tau})=0$, where $\hat{\Omega}_{x}(\hat{\tau})=\left[v_{x}\left(x^{o}(\hat{\tau})\right)-q c_{x}\left(x^{o}(\hat{\tau})\right)-1-\lambda \hat{\tau}\right]$. From (3) evaluated at $\gamma=0$ we derive $\hat{\Omega}_{x}=\tau(1-\lambda)-\left(q-q^{o}\right) c_{x}\left(x^{o}(\tau)\right)$. When $\tau=0, \hat{\Omega}_{\tau}=-\left(q-q^{o}\right) c_{x}\left(x^{o}\right) /\left(v_{x x}\left(x^{o}\right)-q^{o} c_{x x}\left(x^{o}\right)\right)-\lambda x^{o}$, which is positive for all $\lambda<\lambda^{M}$. Moreover, Inada conditions for $v(x)$ together with $c_{x}(0)=0$ imply $\lim _{\tau \rightarrow \infty} x(\tau)=0$. Hence, $\hat{\Omega}_{\tau}<0$ for $\tau$ large enough, and by continuity of $\hat{\Omega}_{\tau}(\tau)$ there exists at least one $\hat{\tau}>0$ satisfying the first-order condition. Finally, $\hat{\Omega}_{\tau \tau}=\left(\hat{\Omega}_{x x} x_{\tau}^{o}-2 \lambda\right) x_{\tau}^{o}+\hat{\Omega}_{x} x_{\tau \tau}^{o}<0$ in $\tau=\hat{\tau}$. Indeed, $x_{\tau \tau}^{o}=-\left(v_{x x x}-q^{o} c_{x x x}\right)\left(x_{\tau}^{o}\right)^{3} \geq 0$ 
since $\left(v_{x x x}-q^{o} c_{x x x}\right) \geq 0$ by assumption, $\hat{\Omega}_{x}(\hat{\tau})<0$ by the first-order condition, and $\hat{\Omega}_{x x} x_{\tau}^{o}-2 \lambda>0$ since $\hat{\Omega}_{x x} x_{\tau}^{o}=\frac{v_{x x}-q c_{x x}}{v_{x x}-q^{o} c_{x x}}>1$ for all $q>q^{o}$, and $\lambda<1 / 2$.

Proof of Proposition 1 Assume $\alpha>0$. First we establish (i). By Kuhn-Tucker necessary conditions, if $\left(\gamma^{*}, \tau^{*}\right)$ maximizes the objective function (4) and $\gamma^{*}<\bar{\gamma}$, then the following equations and inequalities are satisfied:

$$
\begin{cases}\left(\left(v_{x}\left(x^{*}\right)-q c_{x}\left(x^{*}\right)-1-\lambda \tau^{*}\right) x_{\tau}^{o}-\lambda x^{*}\right) \tau^{*}=0 & (K T 1) \\ \left(\left(v_{x}\left(x^{*}\right)-q c_{x}\left(x^{*}\right)-1-\lambda \tau^{*}\right) x_{\gamma}^{o}-b_{\gamma}\right) \gamma^{*}=0 & (K T 2) \\ \left(v_{x}\left(x^{*}\right)-q c_{x}\left(x^{*}\right)-1-\lambda \tau^{*}\right) x_{\tau}^{o}-\lambda x^{*} \leq 0 & (K T 3) \\ \left(v_{x}\left(x^{*}\right)-q c_{x}\left(x^{*}\right)-1-\lambda \tau^{*}\right) x_{\gamma}^{o}-b_{\gamma} \leq 0 & (K T 4) \\ \tau^{*} \geq 0 & \\ \gamma^{*} \geq 0 & \end{cases}
$$

with $x^{*} \equiv x^{o}\left(\gamma^{*}, \tau^{*}\right)$.

From Lemma 1, we know that if $\tau^{*}=0$, then $\gamma^{*}=\hat{\gamma}>0$. To show that whenever $\lambda>0, \gamma^{*}>0$ even if $\tau^{*}>0$, assume $\lambda>0$ and suppose, by way of obtaining a contradiction, that $\gamma^{*}=0$ and $\tau^{*}=\hat{\tau}$ such that (KT3) is satisfied with equality. By substituting $\gamma^{*}=0$ and $\lambda x^{o}(\hat{\tau})=\left(v_{x}\left(x^{o}(\hat{\tau})\right)-q c_{x}\left(x^{o}(\hat{\tau})\right)-1-\lambda \hat{\tau}\right) x_{\tau}^{o}$ in $(K T 4)$, it gives $\lambda x^{o}(\hat{\tau}) q_{\gamma}^{o}(0, \alpha) c_{x}\left(x^{o}(\hat{\tau})\right) \leq b_{\gamma}(0)$, which is a contradiction since $\lambda>0, c_{x}\left(x^{o}(\hat{\tau})\right)>0$, $q_{\gamma}^{o}(0, \alpha)>0$ and $b_{\gamma}(0)=0$ by assumption. Then, $\gamma^{*}>0$ always. Assume now $\lambda>0$. In this case $\tau^{*}=\hat{\tau}^{F B}$ such that $v_{x}\left(x^{*}\right)-q c_{x}\left(x^{*}\right)-1=0$ and $x^{*}=x^{F B}$. By substituting in (KT4), it gives $-b_{\gamma} \leq 0$, which is strictly negative for all $\gamma$. Then, $\gamma^{*}=0$.

Let $\bar{\lambda}=\min \left\{\lambda^{M}, \frac{b_{\gamma}(\hat{\gamma})}{x^{o}(\hat{\gamma}) q_{\gamma}^{o}(\hat{\gamma}, \alpha) c_{x}\left(x^{o}(\hat{\gamma})\right)}\right\}$ and assume $\lambda \in(0, \bar{\lambda})$. This assumption guaranties that the marginal cost of introducing taxation is strictly positive and sufficiently low both with respect to its marginal benefit, and with respect to the marginal cost and benefit of education. We shall prove (ii) and (iii) in 6 steps. The proof relies on Weierstrass theorem arguments since it is difficult to prove that the Hessian is positive definite.

Step 1 If $\lambda<\bar{\lambda}$ then $\tau^{*}>0$.

Assume, by way of obtaining a contradiction, that $\tau^{*}=0$. From Lemma 1, we know that if $\tau^{*}=0$ and $\gamma^{*}=\hat{\gamma} \leq \bar{\gamma}$, then $\left(v_{x}\left(x^{o}(\hat{\gamma})\right)-q c_{x}\left(x^{o}(\hat{\gamma})\right)-1\right) x_{\gamma}^{o}-b_{\gamma} \geq 0$ and $\frac{b_{\gamma}}{q_{\gamma}^{o} c_{x}\left(x^{o}(\hat{\gamma})\right)} \leq\left(v_{x}\left(x^{o}(\hat{\gamma})\right)-q c_{x}\left(x^{o}(\hat{\gamma})\right)-1\right) x_{\tau}^{o}$ since $x_{\gamma}^{o}=x_{\tau}^{o} q_{\gamma}^{o} c_{x}\left(x^{o}(\hat{\gamma})\right)$. By substituting in $(K T 3)$, it gives $\frac{b_{\gamma}(\hat{\gamma})}{q_{\gamma}^{o}(\hat{\gamma}) c_{x}(x(\hat{\gamma}))}-\lambda x(\hat{\gamma}) \leq 0$, which contradicts the assumption $\lambda<\bar{\lambda}$ we have imposed. Then, $\tau^{*}>0$.

Step $2 \Omega_{\tau \gamma}<0$ for all $(\gamma, \tau)$ such that $v_{x}\left(x^{o}(\gamma, \tau)\right)-q c_{x}\left(x^{o}(\gamma, \tau)\right)-1-\lambda \tau<0$.

Let $A \equiv\left\{(\gamma, \tau) \in(0, \hat{\gamma}) \times R_{+}: v_{x}\left(x^{o}(\gamma, \tau)\right)-q c_{x}\left(x^{o}(\gamma, \tau)\right)-1-\lambda \tau<0\right\}$. The cross derivative of $\Omega(\gamma, \tau)$ is

$$
\begin{aligned}
\Omega_{\tau \gamma} & =\left(v_{x x}-q c_{x x}\right) x_{\gamma}^{o} x_{\tau}^{o}+\left(v_{x}-q c_{x}-1-\lambda \tau\right) x_{\gamma \tau}^{o}-\lambda x_{\gamma}^{o} \\
& =\left(\left(v_{x x}-q c_{x x}\right) x_{\tau}^{o}-\lambda\right) x_{\gamma}^{o}+\left(v_{x}-q c_{x}-1-\lambda \tau\right) x_{\gamma \tau}^{o},
\end{aligned}
$$


where $x_{\tau \gamma}^{o}=-\left(x_{\tau}^{o}\right)^{2} q_{\gamma}^{o}\left(\left(v_{x x x}-q^{o}(\gamma) c_{x x x}\right) x_{\tau}^{o} c_{x}-c_{x x}\right)>0$ since $\left(v_{x x x}-q^{o}(\gamma) c_{x x x}\right)>0$ and $c_{x x}$ positive and close to 0 by assumption, and $\left(\left(v_{x x}-q c_{x x}\right) x_{\tau}^{o}-\lambda\right)>0$ since $\left(v_{x x}-q c_{x x}\right) x_{\tau}^{o}=\frac{v_{x x}-q c_{x x}}{v_{x x}-q^{o}(\gamma) c_{x x}}>1$ for all $q^{o}(\gamma)<q$. Hence, $\Omega_{\tau \gamma}<0 \forall(\gamma, \tau) \in A$.

Step 3 There exist $\gamma^{* *} \in(0, \hat{\gamma})$ and $\tau^{* *} \in(0, \hat{\tau})$ such that

$$
\left(\gamma^{* *}, \tau^{* *}\right)=\arg \max \{\Omega(\gamma, \tau): \gamma \in[0, \hat{\gamma}] \text { and } \tau \in[0, \hat{\tau}]\} .
$$

The real function $\Omega(\gamma, \tau)$ is continuous on the non-empty compact space $[0, \hat{\gamma}] \times[0, \hat{\tau}]$, then it attains a global maximum in $[0, \hat{\gamma}] \times[0, \hat{\tau}]$ by Weierstrass' theorem. From point $(i)$ of this proposition and from Step 1, it follows that $\gamma^{* *}>0$ and $\tau^{* *}>0$.

Let $L\left(\gamma, \tau \mu_{1}, \mu_{2}\right)=\Omega(\gamma, \tau)+\mu_{1}(\hat{\tau}-\tau)+\mu_{2}(\hat{\gamma}-\gamma)$ be the Lagrangian function corresponding to the maximization problem (we neglect sign constraints, since we know that $\gamma^{* *}>0$ and $\left.\tau^{* *}>0\right)$. $\left(\gamma^{* *}, \tau^{* *}\right)$ satisfies the following Kuhn-Tucker conditions:

$$
\left\{\begin{array}{l}
\left(v_{x}\left(x^{* *}\right)-q c_{x}\left(x^{* *}\right)-1-\lambda \tau^{* *}\right) x_{\tau}^{o}-\lambda x^{* *}-\mu_{1}=0\left(K T 1^{\prime}\right) \\
\left(v_{x}\left(x^{* *}\right)-q c_{x}\left(x^{* *}\right)-1-\lambda \tau^{* *}\right) x_{\gamma}^{o}-b_{\gamma}-\mu_{2}=0 \quad\left(K T 2^{\prime}\right) \\
\left(\hat{\tau}-\tau^{* *}\right) \mu_{1}=0 \\
\left(\hat{\gamma}-\gamma^{* *}\right) \mu_{2}=0 \\
\hat{\tau} \geq \tau^{* *} ; \mu_{1} \geq 0 \\
\hat{\gamma} \geq \gamma^{* *} ; \mu_{2} \geq 0
\end{array}\right.
$$

with $x^{* *} \equiv x^{o}\left(\gamma^{* *}, \tau^{* *}\right)$. To show that $\tau^{* *}<\hat{\tau}$ assume, by way of obtaining a contradiction, that $\tau^{* *}=\hat{\tau}$. Let $\Omega_{x}(\gamma, \tau)=v_{x}\left(x^{o}(\gamma, \tau)\right)-q c_{x}\left(x^{o}(\gamma, \tau)\right)-1-\lambda \tau$. (KT1') combined with $\mu_{1} \geq 0$ and $x_{\tau}^{o}<0$ imply $\Omega_{\tau}\left(\gamma^{* *}, \hat{\tau}\right)=\Omega_{x}\left(\gamma^{* *}, \hat{\tau}\right) x_{\tau}^{o}-\lambda x^{* *} \geq 0$, $\Omega_{x}\left(\gamma^{* *}, \hat{\tau}\right)<0$ and, then, $\left(\gamma^{* *}, \hat{\tau}\right) \in A$. From Step 2 it follows that $\Omega_{\tau \gamma}\left(\gamma^{* *}, \hat{\tau}\right)<0$. Moreover, since $\Omega_{x \gamma}=\left(v_{x x}-q c_{x x}\right) x_{\gamma}^{o}>0, \Omega_{x}(\gamma, \hat{\tau})<0$ for all $\gamma<\gamma^{* *}$ and, then, $\Omega_{\tau \gamma}(\gamma, \hat{\tau})<0$ for all $\gamma<\gamma^{* *}$. This implies that $\Omega_{\tau}\left(\gamma^{* *}, \hat{\tau}\right)<\Omega_{\tau}(0, \hat{\tau})$. But this contradicts the assumption we started with since, by definition of $\hat{\tau}, \Omega_{\tau}(0, \hat{\tau})=0$. A similar argument can be used to prove that $\gamma^{* *}<\hat{\gamma}$.

Step 4 If $\Omega_{\tau \gamma}\left(\gamma^{\prime}, \tau^{\prime}\right)<0$, then $\Omega_{\tau \gamma}(\gamma, \tau)<0$ for all $\gamma<\gamma^{\prime}$ and $\tau<\tau^{\prime}$.

From Step 2 we know that $\Omega_{\tau \gamma}<0$ whenever $\Omega_{x}<0$. If $\Omega_{x}\left(\gamma^{\prime}, \tau^{\prime}\right)<0$, then $\Omega_{x}(\gamma, \tau)<0$ for all $\gamma<\gamma^{\prime}$ and $\tau<\tau^{\prime}$ since $\Omega_{x \gamma}=\Omega_{x x} x_{\gamma}^{o}>0$ and $\Omega_{x \tau}=\Omega_{x x} x_{\tau}^{o}-\lambda>0$. This implies the claim.

Step 5 If $\left(\gamma^{\prime}, \tau^{\prime}\right)$ solving (8) exists, then it is such that $\gamma^{\prime} \leq \hat{\gamma}$ and $\tau^{\prime} \leq \hat{\tau}$.

Let $\Omega_{\tau}(\gamma, \tau)=\left(v_{x}\left(x^{o}(\gamma, \tau)\right)-q c_{x}\left(x^{o}(\gamma, \tau)\right)-1-\lambda \tau\right) x_{\tau}^{o}-\lambda x^{o}(\gamma, \tau)$. Assume, by way of obtaining a contradiction, that there exist $\tau^{\prime}>\hat{\tau}$ and $\gamma^{\prime}>0$ solving (8). By condition $(K T 1)$ in $(8), \Omega_{\tau}\left(\gamma^{\prime}, \tau^{\prime}\right)=0$ and $\left(\gamma^{\prime}, \tau^{\prime}\right) \in A$.

1. Since $\Omega_{\tau \tau}<0$ for all $(\gamma, \tau) \in A, \Omega_{\tau}\left(\gamma^{\prime}, \hat{\tau}\right)>\Omega_{\tau}\left(\gamma^{\prime}, \tau^{\prime}\right)$.

2. Since $\left(\gamma^{\prime}, \tau^{\prime}\right) \in A, \Omega_{\tau \gamma}\left(\gamma^{\prime}, \tau^{\prime}\right)<0$. From Step $4, \Omega_{\tau \gamma}(\gamma, \tau)<0$ for all $\gamma<\gamma^{\prime}$ and $\tau<\tau^{\prime}$. Hence, $\Omega_{\tau}(0, \hat{\tau})>\Omega_{\tau}\left(\gamma^{\prime}, \hat{\tau}\right)$.

3. By definition of $\hat{\tau}, \Omega_{\tau}(0, \hat{\tau})=0$. 
By combining 1, 2, and 3 one obtains $\Omega_{\tau}\left(\gamma^{\prime}, \tau^{\prime}\right)<0$, which contradicts the assumption we started with. A similar argument can be used to prove that $\gamma^{\prime} \leq \hat{\gamma}$.

Step $\sigma\left(\gamma^{\prime}, \tau^{\prime}\right)$ solving (8) exists and is such that $\gamma^{\prime}=\gamma^{* *}$ and $\tau^{\prime}=\tau^{* *}$ defined in Step 3.

The result follows immediately by combining Step 3 and Step 5.

Assume $\alpha=0$. By substituting it in (KT2), we gets $-b_{\gamma} \gamma=0$, which is true only if $\gamma=0$. The proof is concluded by noting that by Lemma 2, when education is not used, if $\lambda<\lambda^{M}$, the optimal level of taxation is $\hat{\tau}>0$.

Proof of Proposition 2 If the social planner optimization problem defined on page 11 involves an interior solution, $\left(\gamma^{*}, \tau^{*}\right)$ satisfies the following equations:

$$
\left\{\begin{array}{l}
\Omega_{\tau} \equiv\left(v_{x}\left(x^{*}\right)-q c_{x}\left(x^{*}\right)-1-\lambda \tau^{*}\right) x_{\tau}^{o}-\lambda x^{*}=0 \\
\Omega_{\gamma} \equiv\left(v_{x}\left(x^{*}\right)-q c_{x}\left(x^{*}\right)-1-\lambda \tau^{*}\right) x_{\gamma}^{o}-b_{\gamma}=0 .
\end{array}\right.
$$

From the implicit function theorem,

$$
\left(\begin{array}{l}
\partial \tau / \partial \lambda \\
\partial \gamma / \partial \lambda
\end{array}\right)=-\mathbf{H}^{-1} \mathbf{D}_{\lambda}
$$

where $\mathbf{H}^{-1}$ is the inverse of the Hessian matrix of $\Omega(\gamma, \tau)$ evaluated at $\left(\gamma^{*}, \tau^{*}\right)$, that is,

$$
\mathbf{H}^{-\mathbf{1}}=\frac{1}{\Omega_{\tau \tau} \Omega_{\gamma \gamma}-\Omega_{\tau \gamma}^{2}}\left(\begin{array}{cc}
\Omega_{\gamma \gamma} & -\Omega_{\tau \gamma} \\
-\Omega_{\tau \gamma} & \Omega_{\tau \tau}
\end{array}\right),
$$

and $\mathbf{D}_{\lambda}$ is the vector of the first derivatives of $\nabla \Omega$ with respect to $\lambda$, evaluated at $\left(\gamma^{*}, \tau^{*}\right)$, that is,

$$
\mathbf{D}_{\lambda}=\left(\begin{array}{l}
\Omega_{\tau \lambda} \\
\Omega_{\gamma \lambda}
\end{array}\right)=\left(\begin{array}{c}
-\left(\frac{1}{\eta_{x, \tau}}+1\right) \tau x_{\tau}^{o} \\
-\tau x_{\gamma}^{o}
\end{array}\right)=-\tau x_{\tau}^{o}\left(\begin{array}{c}
\left(\frac{1}{\eta_{x, \tau}}+1\right) \\
q_{\gamma}^{o} c_{x}
\end{array}\right)
$$

Substituting in (10), gives

$$
\begin{aligned}
\left(\begin{array}{c}
\partial \tau / \partial \lambda \\
\partial \gamma / \partial \lambda
\end{array}\right) & =\frac{-1}{\Omega_{\tau \tau} \Omega_{\gamma \gamma}-\Omega_{\tau \gamma}^{2}}\left(\begin{array}{cc}
\Omega_{\gamma \gamma} & -\Omega_{\tau \gamma} \\
-\Omega_{\tau \gamma} & \Omega_{\tau \tau}
\end{array}\right)\left(-\tau x_{\tau}^{o}\right)\left(\begin{array}{c}
\left(\frac{1}{\eta_{x, \tau}}+1\right) \\
q_{\gamma}^{o} c_{x}
\end{array}\right) \\
& =\frac{\tau x_{\tau}^{o}}{\Omega_{\tau \tau} \Omega_{\gamma \gamma}-\Omega_{\tau \gamma}^{2}}\left(\begin{array}{c}
\Omega_{\gamma \gamma}\left(\frac{1}{\eta_{x, \tau}}+1\right)-\Omega_{\tau \gamma} q_{\gamma}^{o} c_{x} \\
\Omega_{\tau \tau} q_{\gamma}^{o} c_{x}-\Omega_{\tau \gamma}\left(\frac{1}{\eta_{x, \tau}}+1\right)
\end{array}\right) .
\end{aligned}
$$

From the proof of Proposition 1, we know that $\Omega_{\gamma \tau}<0, \Omega_{\gamma \gamma}<0$, and $\Omega_{\tau \tau}<0$. Moreover, since $\left(\gamma^{*}, \tau^{*}\right)$ maximizes $\Omega$, it follows that when $(\gamma, \tau)=\left(\gamma^{*}, \tau^{*}\right)$ $\Omega_{\tau \tau} \Omega_{\gamma \gamma}-\Omega_{\tau \gamma}^{2} \geq 0$ or, equivalently, $\frac{\Omega_{\gamma \gamma}}{\Omega_{\tau \gamma}} \geq \frac{\Omega_{\tau \gamma}}{\Omega_{\tau \tau}}$. Indeed, if $\Omega_{\tau \tau} \Omega_{\gamma \gamma}-\Omega_{\tau \gamma}^{2}<0,\left(\gamma^{*}, \tau^{*}\right)$ would be a saddle point. Finally, $x_{\tau}^{o}<0, q_{\gamma}^{o} c_{x}>0, \eta_{x, \tau}<0$, and $\left(\frac{1}{\eta_{x, \tau}}+1\right)>0$ iff $\eta_{x, \tau}<-1$. Hence, 


$$
\left\{\begin{array}{l}
\partial \tau / \partial \lambda \geq 0 \text { iff } q_{\gamma}^{o} c_{x} \leq \frac{\Omega_{\gamma \gamma}}{\Omega_{\tau \gamma}}\left(\frac{1}{\eta_{x, \tau}}+1\right) \\
\partial \gamma / \partial \lambda \geq 0 \text { iff } q_{\gamma}^{o} c_{x} \geq \frac{\Omega_{\tau \gamma}}{\Omega_{\tau \tau}}\left(\frac{1}{\eta_{x, \tau}}+1\right)
\end{array}\right.
$$

If $\eta_{x, \tau}>-1$, then $\frac{\Omega_{\gamma \gamma}}{\Omega_{\tau \gamma}}\left(\frac{1}{\eta_{x, \tau}}+1\right)<0$, and $\frac{\Omega_{\tau \gamma}}{\Omega_{\tau \tau}}\left(\frac{1}{\eta_{x, \tau}}+1\right)<0$. Since $q_{\gamma}^{o} c_{x}>0$, this implies $\partial \tau / \partial \lambda \leq 0$ and $\partial \gamma / \partial \lambda \geq 0$.

If $\eta_{x, \tau}<-1$, then $\frac{\Omega_{\gamma \gamma}}{\Omega_{\tau \gamma}}\left(\frac{1}{\eta_{x, \tau}}+1\right) \geq \frac{\Omega_{\tau \gamma}}{\Omega_{\tau \tau}}\left(\frac{1}{\eta_{x, \tau}}+1\right)>0, \quad q_{\gamma}^{o} c_{x} \leq \frac{\Omega_{\gamma \gamma}}{\Omega_{\tau \gamma}}\left(\frac{1}{\eta_{x, \tau}}+1\right) \quad$ iff $\eta_{x, \tau} \leq \bar{\eta} \equiv-\frac{\Omega_{\gamma \gamma}}{\Omega_{\gamma \gamma}-\Omega_{\tau \gamma} q_{\gamma}^{o} c_{x}}$, and $q_{\gamma}^{o} c_{x} \geq \frac{\Omega_{\tau \gamma}}{\Omega_{\tau \tau}}\left(\frac{1}{\eta_{x, \tau}}+1\right)$ iff $\eta_{x, \tau} \geq \underline{\eta} \equiv-\frac{\Omega_{\gamma \tau}}{\Omega_{\gamma \tau}-\Omega_{\tau \tau} q_{\gamma}^{o} c_{x}}$.

To conclude the proof notice that $\underline{\eta}<\bar{\eta}$, since $\Omega_{\tau \tau} \Omega_{\gamma \gamma}-\Omega_{\tau \gamma}^{2} \geq 0$.

Proof of Proposition 3 For all $\gamma \in[0, \bar{\gamma}]$, as long as $v$, and $c$ are thrice differentiable, $\Omega^{h}(\gamma)$ is continuous and twice differentiable. Moreover, $\hat{\Omega}_{\gamma}^{h}=(1-\pi)\left(\mu \hat{\Omega}_{x}^{p} x_{\gamma}^{p}+(1-\mu) \hat{\Omega}_{x}^{o} x_{\gamma}^{o}\right)-b_{\gamma}$, with $\hat{\Omega}_{x}^{a}=\left[v_{x}\left(x^{a}(\gamma)\right)-q c_{x}\left(x^{a}(\gamma)\right)-1\right]$ for any $a \in\{o, p\}$. From (5), we can derive $\hat{\Omega}_{x}^{o}(\gamma)=-(\bar{\delta}-\delta(\gamma)) c_{x}\left(x^{o}(\gamma)\right)<0$, and $\hat{\Omega}_{x}^{p}(\gamma)=(\bar{\delta}-\delta(\gamma)) c_{x}\left(x^{p}(\gamma)\right)>0$. Moreover, $x_{\gamma}^{o}=\frac{\delta_{\gamma} c_{c}(x)}{v_{x x}(x)-q^{o}(\gamma) c_{x x}(x)}<0$ and $x_{\gamma}^{p}=\frac{-\delta_{\gamma} c_{x}(x)}{v_{x x}(x)-q^{o}(\gamma) c_{x x}(x)}>0$ since $q_{\gamma}^{o}=\delta_{\gamma}>0, q_{\gamma}^{p}=-\delta_{\gamma}<0, c_{x}>0$, and $v_{x x}-q^{a} c_{x x}<0$ for any $a \in\{o, p\}$ by assumption. Hence, both $\hat{\Omega}_{x}^{p} x_{\gamma}^{p}$ and $\hat{\Omega}_{x}^{o} x_{\gamma}^{o}$ are positive.

When $\quad \gamma=0, \quad \hat{\Omega}_{\gamma}^{h}=(1-\pi)\left(\mu \bar{\delta} c_{x}\left(x^{p}\right) x_{\gamma}^{p}-(1-\mu) \bar{\delta} c_{x}\left(x^{o}\right) x_{\gamma}^{o}\right)>0 . \quad$ Moreover, $q^{p}(\bar{\gamma})=q^{o}(\bar{\gamma})=q$ implies that when $\gamma=\bar{\gamma}, \hat{\Omega}_{x}^{a}(\bar{\gamma})=0$ for any $a \in\{o, p\}$ and $\hat{\Omega}_{\gamma}^{h}=-b_{\gamma}(\bar{\gamma})<0$. Hence, $\gamma=0$ and $\gamma=\bar{\gamma}$ cannot be corner solutions of the social planner maximization problem and there exists $\hat{\gamma}^{h} \in(0, \bar{\gamma})$ satisfying condition $\hat{\Omega}_{\gamma}^{h}\left(\hat{\gamma}^{h}\right)=0$.

Assume first $\mu=0$. In this case, the optimal level of education provision is $\hat{\gamma}^{h^{\prime}}$ such that $\hat{\Omega}_{\gamma}^{h^{\prime}} \equiv(1-\pi) \hat{\Omega}_{x}^{o} x_{\gamma}^{o}-b_{\gamma}=0 \cdot \hat{\gamma}^{h^{\prime}}$ is unique since $\hat{\Omega}_{\gamma \gamma}^{h^{\prime}}=(1-\pi)\left[\hat{\Omega}_{x}^{o} x_{\gamma \gamma}^{o}+\hat{\Omega}_{x x}^{o} x_{\gamma}^{o 2}\right]-b_{\gamma \gamma}<0$ from the proof of Lemma 1 , and it is lower than $\hat{\gamma}$ since $\hat{\Omega}_{\gamma}^{h^{\prime}}=0 \Leftrightarrow \hat{\Omega}_{\gamma}=\pi \hat{\Omega}_{x}^{o} x_{\gamma}^{o}$, $\hat{\Omega}_{\gamma \gamma}<0$ and $\hat{\Omega}_{x}^{o} x_{\gamma}^{o}>0$ from the proof of Lemma 1 .

Assume now $\mu \geq 0$. From the implicit function theorem, into a neighborhood of $\hat{\gamma}^{h}=\hat{\gamma}^{h^{\prime}}$ and $\mu=0$,

$$
\partial \hat{\gamma}^{h} / \partial \mu=-\frac{(1-\pi)\left(\bar{\delta}-\delta\left(\hat{\gamma}^{h^{\prime}}\right)\right)\left(c_{x}\left(x^{p}\left(\hat{\gamma}^{h^{\prime}}\right)\right) x_{\gamma}^{p}\left(x^{p}\left(\hat{\gamma}^{h^{\prime}}\right)\right)+c_{x}\left(x^{o}\left(\hat{\gamma}^{h^{\prime}}\right)\right) x_{\gamma}^{o}\left(x^{o}\left(\hat{\gamma}^{h^{\prime}}\right)\right)\right)}{\hat{\Omega}_{\gamma \gamma}^{h}},
$$

which is lower than zero iff $c_{x}\left(x^{p}\left(\hat{\gamma}^{h^{\prime}}\right)\right) x_{\gamma}^{p}\left(x^{p}\left(\hat{\gamma}^{h^{\prime}}\right)\right)<-c_{x}\left(x^{o}\left(\hat{\gamma}^{h^{\prime}}\right)\right) x_{\gamma}^{o}\left(x^{o}\left(\hat{\gamma}^{h^{\prime}}\right)\right)$, that is always true if $c_{x x}$ is positive and small enough. Indeed, $c_{x}\left(x^{p}\left(\hat{\gamma}^{h^{\prime}}\right)\right)<c_{x}\left(x^{o}\left(\hat{\gamma}^{h^{\prime}}\right)\right)$ since $c_{x x}>0$ and $x^{p}\left(\hat{\gamma}^{h^{\prime}}\right)<x^{o}\left(\hat{\gamma}^{h^{\prime}}\right)$. Moreover, if the health cost function is not too convex, then the concavity of the consumer's utility functions implies $\left|x_{\gamma}^{o}(\gamma)\right| \geq\left|x_{\gamma}^{p}(\gamma)\right|$ for any $\gamma$. Indeed, the effectiveness of information provision of optimistic and pessimistic agents differ for two reasons. On the one hand, for any level of sin good consumption $\quad x, \quad \frac{\delta_{\gamma} c_{x}(x)}{\left|v_{x x}(x)-q^{o}(\gamma) c_{x x}(x)\right|}>\frac{\delta_{\gamma} c_{x}(x)}{\left|v_{x x}(x)-q^{p}(\gamma) c_{x x}(x)\right|} \quad$ since $\quad q^{p}>q^{o} \quad$ and $v_{x x}(x)-q^{p}(\gamma) c_{x x}(x)<v_{x x}(x)-q^{o}(\gamma) c_{x x}(x)<0$. On the other hand, both $\frac{\delta_{\gamma} c_{x}(x)}{\left|v_{x x}(x)-q^{o}(\gamma) c_{x x}(x)\right|}$ and 
$\frac{\delta_{\gamma} c_{x}(x)}{\left|v_{x x}(x)-q^{p}(\gamma) c_{x x}(x)\right|}$ are increasing in $x$ and $x^{o}(\gamma)>x^{p}(\gamma)$. If $c_{x x}$ is small, the second effect prevails on the first. As a consequence, pessimistic consumers lower the social planner's marginal benefit of information provision and reduce the optimal $\gamma$ for any $\pi \geqq 0$. In particular, if $\pi=0, \quad \partial \hat{\gamma}^{h} / \partial \mu<0$ and then $\hat{\gamma}^{\pi=0}$ such that $\hat{\Omega}_{\gamma}^{\pi=0} \equiv\left(\mu \hat{\Omega}_{x}^{p} x_{\gamma}^{p}+(1-\mu) \hat{\Omega}_{x}^{o} x_{\gamma}^{o}\right)-b_{\gamma}=0$ exists and is lower than $\hat{\gamma}$.

Finally, from the implicit function theorem,

$$
\frac{\partial \hat{\gamma}^{h}}{\partial \pi}=\frac{\left(\mu \hat{\Omega}_{x}^{p} x_{\gamma}^{p}+(1-\mu) \hat{\Omega}_{x}^{o} x_{\gamma}^{o}\right)}{\hat{\Omega}_{\gamma \gamma}^{h}},
$$

which is lower than zero since at the optimum $\left(\mu \hat{\Omega}_{x}^{p} x_{\gamma}^{p}+(1-\mu) \hat{\Omega}_{x}^{o} x_{\gamma}^{o}\right)=\frac{b_{\gamma}\left(\hat{\gamma}^{h}\right)}{1-\pi}>0$.

Lemma 3 Assume $\mu=0$ and $\lambda<\lambda^{M^{h}}$, with $\lambda^{M^{h}}=\min \left\{\frac{(1-\pi)\left(q-q^{o}\right)\left|c_{\tau}\left(x^{o}\right)\right|}{\pi x^{F B}+(1-\pi) x^{o}}, \frac{1}{2}\right\}$. When education is not used, the optimal level of taxation is $\hat{\tau}^{h^{\prime}}>0$ such that $\hat{\Omega}_{\tau}^{h^{\prime}}\left(\hat{\tau}^{h^{\prime}}\right)=0$, where $\hat{\Omega}^{h^{\prime}}(\tau) \equiv B^{h}(\gamma=0, \tau ; \mu=0)-C T^{h}(\gamma=0, \tau ; \mu=0)$.

Proof of Lemma 3 When a linear tax $\tau$ and lump sum transfer $l$ are introduced, the optimal consumption of a type $a$ agent maximizes the expected utility $U^{a}$, subject to the budget constraint $z=I+l-(1+\tau) x$. Then, the sin good consumption rule of an unbiased agent, $x^{u}(\tau)$, satisfies the first-order condition

$$
v_{x}(x)-q c_{x}(x)=(1+\tau),
$$

and the sin good consumption rule of an optimistic agent, $x^{o}(\tau)$, satisfies the firstorder condition

$$
v_{x}(x)-q^{o}(\gamma, \alpha) c_{x}(x)=(1+\tau) .
$$

For all $\tau \geq 0$, as long as $v$ and $c$ are thrice differentiable, $\hat{\Omega}^{h^{\prime}}(\tau)$ is continuous and twice differentiable. If strictly positive, $\hat{\tau}^{h^{\prime}}$ satisfies the first-order condition $\hat{\Omega}_{\tau}^{h^{\prime}}\left(\hat{\tau}^{h^{\prime}}\right)=\pi\left(\hat{\Omega}_{x}^{u}\left(\hat{\tau}^{h^{\prime}}\right) x_{\tau}^{u}\left(\hat{\tau}^{h^{\prime}}\right)-\lambda x^{u}\left(\hat{\tau}^{h^{\prime}}\right)\right)+(1-\pi)\left(\hat{\Omega}_{x}^{o}\left(\hat{\tau}^{h^{\prime}}\right) x_{\tau}^{o}(\hat{\tau})-\lambda x^{o}\left(\hat{\tau}^{h^{\prime}}\right)\right)=0 \quad$, where $\hat{\Omega}_{x}^{a}\left(\hat{\tau}^{h^{\prime}}\right)=\left[v_{x}\left(x^{a}\left(\hat{\tau}^{h^{\prime}}\right)\right)-q c_{x}\left(x^{a}\left(\hat{\tau}^{h^{\prime}}\right)\right)-1-\lambda \hat{\tau}^{h^{\prime}}\right]$, for all $a \in\{u, o\}$. From (11) we derive $\hat{\Omega}_{x}^{u}=\tau(1-\lambda)$. From (12) we derive $\hat{\Omega}_{x}^{o}=\tau(1-\lambda)-\left(q-q^{o}\right) c_{x}\left(x^{o}(\tau)\right)$. When $\tau=0, \quad \hat{\Omega}_{\tau}^{h^{\prime}}=-\pi \lambda x^{F B}-(1-\pi)\left(\left(q-q^{o}\right) c_{x}\left(x^{o}\right) /\left(v_{x x}\left(x^{o}\right)-q^{o} c_{x x}\left(x^{o}\right)\right)+\lambda x^{o}\right)$, which is positive for all $\lambda<\lambda^{M^{h}}$. Moreover, Inada conditions for $v(x)$ together with $c_{x}(0)=0$ imply $\lim _{\tau \rightarrow \infty} x^{i}(\tau)=0$, for all $i \in\{u, o\}$. Hence, $\hat{\Omega}_{\tau}^{h^{\prime}}<0$ for $\tau$ large enough and, by continuity of $\hat{\Omega}_{\tau}^{h^{\prime}}(\tau)$, there exists at least one $\hat{\tau}^{h^{\prime}}>0$ satisfying the first-order condition.

Proof of Proposition 4 Denote with $\left(\gamma^{h \prime}, \tau^{h \prime}\right) \equiv\left(\gamma_{\mid \mu=0}^{h^{*}}, \tau_{\mid \mu=0}^{h^{*}}\right)$ the optimal policy given $\mu=0$. The following Kuhn-Tucker necessary conditions are satisfied: 


$$
\left\{\begin{array}{l}
{\left[\pi \Omega_{\tau}^{u}\left(\gamma^{h \prime}, \tau^{h \prime}\right)+(1-\pi) \Omega_{\tau}^{o}\left(\gamma^{h \prime}, \tau^{h \prime}\right)\right] \tau^{h \prime}=0\left(K T 1^{h^{\prime}}\right)} \\
{\left[\pi \Omega_{\gamma}^{u}\left(\gamma^{h \prime}, \tau^{h \prime}\right)+(1-\pi) \Omega_{\gamma}^{o}\left(\gamma^{h \prime}, \tau^{h \prime}\right)\right] \gamma^{h \prime}=0\left(K T 2^{h^{\prime}}\right)} \\
\pi \Omega_{\tau}^{u}\left(\gamma^{h \prime}, \tau^{h \prime}\right)+(1-\pi) \Omega_{\tau}^{o}\left(\gamma^{h \prime}, \tau^{h \prime}\right) \leq 0\left(K T 3^{h^{\prime}}\right) \\
\pi \Omega_{\gamma}^{u}\left(\gamma^{h \prime}, \tau^{h \prime}\right)+(1-\pi) \Omega_{\gamma}^{o}\left(\gamma^{h \prime}, \tau^{h \prime}\right) \leq 0\left(K T 4^{h^{\prime}}\right) \\
\tau^{h \prime} \geq 0 \\
\gamma^{h \prime} \geq 0
\end{array}\right.
$$

with $\quad \Omega_{\tau}^{a}\left(\gamma^{h \prime}, \tau^{h \prime}\right) \equiv \Omega_{x}^{a}\left(\gamma^{h \prime}, \tau^{h \prime}\right) x_{\tau}^{a}-\lambda x^{a^{\prime}}, \quad \Omega_{\gamma}^{a}\left(\gamma^{h^{\prime}}, \tau^{h^{\prime}}\right) \equiv \Omega_{x}^{a}\left(\gamma^{h \prime}, \tau^{h \prime}\right) x_{\gamma}^{a^{\prime}}-b_{\gamma}$, $\Omega_{x}^{a}\left(\gamma^{h^{\prime}}, \tau^{\tau}\right) \equiv\left(v_{x}\left(x^{a^{\prime}}\right)-q c_{x}\left(x^{a^{\prime}}\right)-1^{\tau}-\lambda \tau^{h^{\prime}}\right), \quad$ and,$x^{a^{\prime}} \equiv x^{a}\left(\gamma^{h^{\prime}}, \tau^{h^{\prime}}\right)$ for $\quad$ any $a \in\{u, o\}$. Since $x_{\gamma}^{u}=0$ implies $\Omega_{\gamma}^{u}=-b_{\gamma}$, then $\left(K T 4^{h^{\prime}}\right)$ can be rewritten as:

$$
\left.l r-\pi b_{\gamma}+(1-\pi) \Omega_{\gamma}^{o}\left(\gamma^{h \prime}, \tau^{h \prime}\right)\right] \gamma^{h \prime}=0 .\left(K T 4^{h^{\prime}}\right)^{\prime}
$$

From Lemma 3, we know that if $\tau^{h^{\prime}}=0$, then $\gamma^{h^{\prime}}=\hat{\gamma}^{\mu=0}>0$. To show that $\gamma^{h^{\prime}}>0$ even if $\tau^{h^{\prime}}>0$ suppose, by way of obtaining a contradiction, that $\gamma^{h^{\prime}}=0$ and $\tau^{h^{\prime}}=\hat{\tau}^{\mu=0}$ such that $\left(K T 3^{h^{\prime}}\right)$ is satisfied with equality. By substituting $\gamma^{h^{\prime}}=0$ and $\tau^{h^{\prime}}=\hat{\tau}^{\mu=0}$ in $\left(K T 2^{h^{\prime}}\right)^{\prime}$ one gets $(1-\pi) \Omega_{x}^{o}\left(\gamma^{h \prime}, \tau^{h \prime}\right) x_{\gamma}^{o} \leq 0$, which is a contradiction since $x_{\gamma}^{o}<0$ and $\Omega_{x}^{o}\left(\gamma^{h \prime}, \tau^{h \prime}\right)<0$. Indeed, by combining $\left(K T 3^{h^{\prime}}\right)$ and $\hat{\tau}^{h^{\prime}}>0$ we obtain $\pi \Omega_{\tau}^{u}\left(\gamma^{h \prime}, \tau^{h \prime}\right)+(1-\pi) \Omega_{\tau}^{o}\left(\gamma^{h \prime}, \tau^{h \prime}\right)=0$, that implies $\pi \Omega_{x}^{o}\left(\gamma^{h \prime}, \tau^{h \prime}\right) x_{\tau}^{o}+(1-\pi) \Omega_{x}^{u}\left(\gamma^{h \prime}, \tau^{h \prime}\right) x_{\tau}^{u}=\lambda\left(\pi x^{o^{\prime}}+(1-\pi) x^{u^{\prime}}\right)>0$, which can be true only if $\inf \left\{\Omega_{x}^{u}\left(\gamma^{h \prime}, \tau^{h \prime}\right), \Omega_{x}^{o}\left(\gamma^{h \prime}, \tau^{h \prime}\right)\right\}=\Omega_{x}^{o}\left(\gamma^{h \prime}, \tau^{h \prime}\right)<0$ since both $x_{\tau}^{u}<0$ and $x_{\tau}^{o}<0$. Then, $\gamma^{h \prime}>0$ always.

Let $\quad \bar{\lambda}^{h}=\min \left\{\lambda^{M^{h}}, \frac{b_{\gamma}}{\left[\pi x^{F B}+(1-\pi) x\left(\hat{\gamma}^{\mu=0}\right)\right] q_{\gamma}^{o} c_{x}\left(x\left(\hat{\gamma}^{\mu=0}\right)\right)}\right\} \quad$ and assume $\lambda<\bar{\lambda}^{h}$. This assumption guaranties that the exogenous marginal cost of introducing taxation is sufficiently low both with respect to its marginal benefit, and with respect to the marginal cost and benefit of education. To prove that if $\lambda<\bar{\lambda}$ then $\tau^{h \prime}>0$, assume, by way of obtaining a contradiction, that $\tau^{h \prime}=0$. From Proposition 3, we know that if $\tau^{h^{\prime}}=0$ and $\gamma^{h^{\prime}}=\hat{\gamma}^{h^{\prime}} \leq \bar{\gamma}$, then $(1-\pi)\left(v_{x}\left(x^{o}\left(\hat{\gamma}^{\prime}\right)\right)-q c_{x}\left(x^{o}\left(\hat{\gamma}^{h^{\prime}}\right)\right)-1\right) x_{\gamma}^{o}-b_{\gamma} \geq 0$ and $\quad \frac{b_{\gamma}}{q_{p}^{o} c_{x}\left(x^{\prime}\left(\hat{\gamma}^{\prime \prime}\right)\right)} \leq(1-\pi)\left(v_{x}\left(x^{o}\left(\hat{\gamma}^{h^{\prime}}\right)\right)-q c_{x}\left(x^{o}\left(\hat{\gamma}^{h^{\prime}}\right)\right)-1\right) x_{\tau}^{o} \quad$ since $\quad x^{o^{\prime}}=x^{o}\left(\hat{\gamma}^{h^{\prime}}\right) \quad$ and $x_{\gamma}^{o}=x_{\tau}^{o} q_{\gamma}^{o} c_{x}\left(x^{o}\left(\hat{\gamma}^{h^{\prime}}\right)\right)$, and $\left(v_{x}\left(x^{u}\right)-q c_{x}\left(x^{u}\right)-1\right)=0$ since $x^{u}=x^{F B}$. By substituting in $\left(K T 3^{h^{\prime}}\right)$, one gets $\frac{b_{\gamma}\left(\hat{\gamma}^{h^{\prime}}\right)}{q_{\gamma}^{o} c_{x}\left(x\left(\hat{\gamma}^{h^{\prime}}\right)\right)}-\lambda\left[\pi x^{F B}+(1-\pi) x\left(\hat{\gamma}^{h^{\prime}}\right)\right] \leq 0$, which contradicts the assumption $\lambda<\bar{\lambda}^{h}$ we have imposed. Then, $\tau^{h^{\prime}}>0$.

Finally, $\lim _{\pi \rightarrow 1} \bar{\lambda}^{h} \leq \lim _{\pi \rightarrow 1} \lambda^{M^{h}}=0$.

Proof of Proposition 5 Assume $\lambda<\bar{\lambda}^{h}$. If $\left(\gamma^{h}, \tau^{h}\right)>0$ maximize $\Omega^{h}(\gamma, \tau) \equiv B^{h}(\gamma, \tau)-C T^{h}(\gamma, \tau)$ and $\gamma^{h}<\bar{\gamma}$, then by Kuhn-Tucker necessary conditions the following equations are satisfied:

$$
\left\{\begin{array}{l}
\pi \Omega_{\tau}^{u}\left(\gamma^{h}, \tau^{h}\right)+(1-\pi)\left[(1-\mu) \Omega_{\tau}^{o}\left(\gamma^{h}, \tau^{h}\right)+\mu \Omega_{\tau}^{p}\left(\gamma^{h}, \tau^{h}\right)\right]=0\left(K T 1^{h}\right) \\
\pi \Omega_{\gamma}^{u}\left(\gamma^{h}, \tau^{h}\right)+(1-\pi)\left[(1-\mu) \Omega_{\gamma}^{o}\left(\gamma^{h}, \tau^{h}\right)+\mu \Omega_{\gamma}^{p}\left(\gamma^{h}, \tau^{h}\right)\right]=0\left(K T 2^{h}\right)
\end{array}\right.
$$


with $\quad \Omega_{\tau}^{a}\left(\gamma^{h}, \tau^{h}\right) \equiv \Omega_{x}^{a}\left(\gamma^{h}, \tau^{h}\right) x_{\tau}^{a}-\lambda x^{a^{h}}, \quad \Omega_{\gamma}^{a}\left(\gamma^{h}, \tau^{h}\right) \equiv \Omega_{x}^{a}\left(\gamma^{h}, \tau^{h}\right) x_{\gamma}^{a}-b_{\gamma}$, $\Omega_{x}^{a}\left(\gamma^{h}, \tau^{h}\right) \equiv\left(v_{x}\left(x^{a^{h}}\right)-q c_{x}\left(x^{a^{h}}\right)-1-\lambda \tau^{h}\right), \quad$ and $x^{a^{h}} \equiv x^{a^{h}}\left(\gamma^{h}, \tau^{h}\right)$ for any $a \in\{u, o, p\}$, and by the second-order conditions for a maximum, $\Omega_{\gamma \gamma}^{h}\left(\gamma^{h}, \tau^{h}\right)<0$, $\Omega_{\tau \tau}^{h}\left(\gamma^{h}, \tau^{h}\right)<0$ and $\Omega_{\tau \tau}\left(\gamma^{h}, \tau^{h}\right) \Omega_{\gamma \gamma}\left(\gamma^{h}, \tau^{h}\right)-\Omega_{\tau \gamma}^{2}\left(\gamma^{h}, \tau^{h}\right)^{2} \geq 0$. Since $x_{\gamma}^{u^{h}}=0$ implies $\Omega_{\gamma}^{u}\left(\gamma^{h}, \tau^{h}\right)=-b_{\gamma}$, then $\left(K T 2^{h}\right)$ can be rewritten as:

$$
-\pi b_{\gamma}+(1-\pi)\left[(1-\mu) \Omega_{\gamma}^{o}\left(\gamma^{h}, \tau^{h}\right)+\mu \Omega_{\gamma}^{p}\left(\gamma^{h}, \tau^{h}\right)\right]=0 .\left(K T 2^{h}\right)^{\prime}
$$

Let $\Omega^{\mu}(\gamma, \tau) \equiv \Omega(\gamma, \tau, \mu)$ and notice that when $\mu=0$ the optimal policy pair is $\left(\gamma^{h}, \tau^{h}\right)=\left(\gamma^{h \prime}, \tau^{h^{\prime}}\right)$ defined in Proposition 4 . We will prove the proposition in 3 steps.

Step $1 \Omega_{\tau \mu}\left(\gamma^{h \prime}, \tau^{h^{\prime}}, 0\right)<0$. The derivative of $\Omega_{\tau}(\gamma, \tau, \mu)$ with respect to $\mu$ in $\mu=0$ is:

$$
\begin{aligned}
\Omega_{\tau \mu}\left(\gamma^{h \prime}, \tau^{h^{\prime}}, 0\right) \equiv & (1-\pi)\left[\Omega_{\tau}^{p}\left(\gamma^{h \prime}, \tau^{h^{\prime}}\right)-\Omega_{\tau}^{o}\left(\gamma^{h \prime}, \tau^{h^{\prime}}\right)\right] \\
= & \pi\left[\Omega_{x}^{u}\left(\gamma^{h \prime}, \tau^{h^{\prime}}\right) x_{\tau}^{u}-\lambda x^{u}\left(\gamma^{h \prime}, \tau^{h^{\prime}}\right)\right] \\
& +(1-\pi)\left[\Omega_{x}^{p}\left(\gamma^{h \prime}, \tau^{h^{\prime}}\right) x_{\tau}^{p}-\lambda x^{p}\left(\gamma^{h \prime}, \tau^{h^{\prime}}\right)\right],
\end{aligned}
$$

since $-(1-\pi) \Omega_{\tau}^{o}\left(\gamma^{h \prime}, \tau^{h^{\prime}}\right)=\pi \Omega_{\tau}^{u}\left(\gamma^{h \prime}, \tau^{h^{\prime}}\right)$ by definition of $\left(\gamma^{h \prime}, \tau^{h^{\prime}}\right)$. Notice that: (1) $\Omega_{x}^{u}\left(\gamma^{h \prime}, \tau^{h^{\prime}}\right)=(1-\lambda) \tau^{h^{\prime}}>0$ and $(2) \Omega_{x}^{p}=\left(\left(q^{p}\left(\gamma^{h^{\prime}}\right)-q\right) c_{x}\left(x^{a}\left(\gamma^{h \prime}, \tau^{h^{\prime}}\right)\right)+(1-\lambda) \tau^{h^{\prime}}\right)>0$ since $q^{p}\left(\gamma^{h^{\prime}}\right)>q$. By combining (1) and (2) with $x_{\tau}^{u}<0$ and $x_{\tau}^{p}<0$, one gets $\Omega_{\tau \mu}\left(\gamma^{h \prime}, \tau^{h^{\prime}}, 0\right)<0$.

Step $2 \Omega_{\gamma \mu}\left(\gamma^{h \prime}, \tau^{h^{\prime}}, 0\right)>0$. The derivative of $\Omega_{\gamma}(\gamma, \tau, \mu)$ with respect to $\mu$ in $\mu=0$ is:

$$
\begin{aligned}
\Omega_{\gamma \mu}\left(\gamma^{h \prime}, \tau^{h^{\prime}}, 0\right) \equiv & (1-\pi)\left[\Omega_{\gamma}^{p}\left(\gamma^{h \prime}, \tau^{h^{\prime}}\right)-\Omega_{\gamma}^{o}\left(\gamma^{h \prime}, \tau^{h^{\prime}}\right)\right] \\
= & (1-\pi)\left[\frac{-\Omega_{x}^{p} \delta_{\gamma}\left(\gamma^{h^{\prime}}\right) c_{x}\left(x^{p}\left(\gamma^{h \prime}, \tau^{h^{\prime}}\right)\right)}{v_{x x}\left(x^{p}\left(\gamma^{h \prime}, \tau^{h^{\prime}}\right)\right)-q^{p}\left(\gamma^{*}\right) c_{x x}\left(x^{p}\left(\gamma^{h \prime}, \tau^{h^{\prime}}\right)\right)}\right. \\
& \left.-\frac{\Omega_{x}^{o} \delta_{\gamma}\left(\gamma^{h^{\prime}}\right) c_{x}\left(x^{o}\left(\gamma^{h \prime}, \tau^{h^{\prime}}\right)\right)}{v_{x x}\left(x^{o}\left(\gamma^{h \prime}, \tau^{h^{\prime}}\right)\right)-q^{o}\left(\gamma^{h^{\prime}}\right) c_{x x}\left(x^{o}\left(\gamma^{h \prime}, \tau^{h^{\prime}}\right)\right)}\right],
\end{aligned}
$$

which is higher than zero iff

$$
\frac{v_{x x}\left(x^{p}\left(\gamma^{h \prime}, \tau^{h^{\prime}}\right)\right)-q^{p}\left(\gamma^{h^{\prime}}\right) c_{x x}\left(x^{p}\left(\gamma^{h \prime}, \tau^{h^{\prime}}\right)\right)}{v_{x x}\left(x^{o}\left(\gamma^{h \prime}, \tau^{h^{\prime}}\right)\right)-q^{o}\left(\gamma^{h^{\prime}}\right) c_{x x}\left(x^{o}\left(\gamma^{h \prime}, \tau^{h^{\prime}}\right)\right)}<\frac{c_{x}\left(x^{p}\left(\gamma^{h \prime}, \tau^{h^{\prime}}\right)\right)}{c_{x}\left(x^{o}\left(\gamma^{h \prime}, \tau^{h^{\prime}}\right)\right)} \frac{\Omega_{x}^{p}}{-\Omega_{x}^{o}} .
$$

The left-hand side of (14) is lower than 1 since $\frac{\partial\left(v_{x x}-q c_{x x}\right)}{\partial q}=\left(v_{x x x}-q c_{x x x}\right) x_{q}-c_{x x}<0$. The right-hand side of (14) is higher than 1 since $c_{x x}$ is near to 0 by assumption and then the distance between $c_{x}\left(x^{p}\left(\gamma^{h \prime}, \tau^{h^{\prime}}\right)\right)$ and $c_{x}\left(x^{o}\left(\gamma^{h \prime}, \tau^{h^{\prime}}\right)\right)$ is small. Indeed, if $\left|c_{x}\left(x^{p}\left(\gamma^{h \prime}, \tau^{h^{\prime}}\right)\right)-c_{x}\left(x^{o}\left(\gamma^{h \prime}, \tau^{h^{\prime}}\right)\right)\right|<\epsilon$, with $\epsilon>0$ and small enough, $\frac{c_{x}\left(x^{p}\left(\gamma^{h \prime}, \tau^{h^{\prime}}\right)\right)}{c_{x}\left(x^{o}\left(\gamma^{h \prime}, \tau^{h^{\prime}}\right)\right)}$ is near to 1 and 


$$
\frac{\Omega_{x}^{p}}{-\Omega_{x}^{o}}=\frac{\left(\bar{\delta}-\delta\left(\gamma^{h^{\prime}}\right)\right) c_{x}\left(x^{p}\left(\gamma^{h^{\prime}}, \tau^{h^{\prime}}\right)\right)+(1-\lambda) \tau^{h^{\prime}}}{\left(\bar{\delta}-\delta\left(\gamma^{h^{\prime}}\right)\right) c_{x}\left(x^{o}\left(\gamma^{h \prime}, \tau^{h^{\prime}}\right)\right)-(1-\lambda) \tau^{h^{\prime}}}>1
$$

since $\Omega_{x}^{a}=\left(\left(q^{a}\left(\gamma^{h^{\prime}}\right)-q\right) c_{x}\left(x^{a}\left(\gamma^{h^{\prime}}, \tau^{h^{\prime}}\right)\right)+(1-\lambda) \tau^{h^{\prime}}\right)$ for any $a \in\{o, p\}$, and $2(1-\lambda) \tau^{h^{\prime}}>\left((\bar{\delta}-\delta(\gamma))\left(c_{x}\left(x^{o}\left(\gamma^{h^{\prime}}, \tau^{h^{\prime}}\right)\right)-c_{x}\left(x^{p}\left(\gamma^{h \prime}, \tau^{h^{\prime}}\right)\right)\right)\right.$.

Step $3 \tau^{h}<\tau^{h^{\prime}}$ and $\gamma^{h^{*}}>\gamma^{h^{\prime}}$. Assume $\Omega_{\tau \tau}\left(\gamma^{h}, \tau^{h}\right) \Omega_{\gamma \gamma}\left(\gamma^{h}, \tau^{h}\right)-\Omega_{\tau \gamma}^{2}\left(\gamma^{h}, \tau^{h}\right)^{2}>0$. The implicit function theorem into a neighborhood of $\gamma=\gamma^{h^{\prime}}, \tau=\tau^{h^{\prime}}$, and $\mu=0$ gives:

$$
\left(\begin{array}{c}
\partial \tau^{h} / \partial \mu \\
\partial \gamma^{h} / \partial \mu
\end{array}\right)=-\left(\mathbf{H}^{\mathbf{h}}\right)^{-1} \mathbf{D}_{\boldsymbol{\mu}}^{\mathbf{h}}
$$

where $\left(\mathbf{H}^{\mathbf{h}}\right)^{\mathbf{- 1}}$ is the inverse of the Hessian matrix of $\Omega(\gamma, \tau, \mu)$ evaluated at $\gamma=\gamma^{h^{\prime}}$, $\tau=\tau^{h^{\prime}}$, and $\mu=0$, that is,

$$
\left(\mathbf{H}^{\mathbf{h}}\right)^{-\mathbf{1}}=\Psi\left(\begin{array}{cc}
\Omega_{\gamma \gamma}\left(\gamma^{h^{\prime}}, \tau^{h^{\prime}}, 0\right) & -\Omega_{\tau \gamma}\left(\gamma^{h^{\prime}}, \tau^{h^{\prime}}, 0\right) \\
-\Omega_{\tau \gamma}\left(\gamma^{h^{\prime}}, \tau^{h^{\prime}}, 0\right) & \Omega_{\tau \tau}\left(\gamma^{h^{\prime}}, \tau^{h^{\prime}}, 0\right)
\end{array}\right),
$$

where $\Psi \equiv \frac{1}{\Omega_{\tau \tau}\left(\gamma^{h^{\prime}}, \tau^{h^{\prime}}, 0\right) \Omega_{\gamma \gamma}\left(\gamma^{h^{\prime}}, \tau^{h^{\prime}}, 0\right)-\Omega_{\tau \gamma}\left(\gamma^{h^{\prime}}, \tau^{h^{\prime}}, 0\right)}$, , and $\mathbf{D}_{\boldsymbol{\mu}}^{\mathbf{h}}$ is the vector of the first derivatives of $\nabla \Omega^{h}$ with respect to $\mu$, evaluated at $\left(\gamma^{h^{\prime}}, \tau^{h^{\prime}}, 0\right)$, that is,

$$
\mathbf{D}_{\boldsymbol{\mu}}^{\mathbf{h}}=\left(\begin{array}{c}
\Omega_{\tau \mu}\left(\gamma^{h^{\prime}}, \tau^{h^{\prime}}, 0\right) \\
\Omega_{\gamma \mu}\left(\gamma^{h^{\prime}}, \tau^{h^{\prime}}, 0\right)
\end{array}\right)
$$

Substituting in (15), gives

$$
\left\{\begin{array}{l}
\partial \tau^{h} / \partial \mu \propto \Omega_{\tau \gamma} \Omega_{\gamma \mu}\left(\gamma^{h^{\prime}}, \tau^{h^{\prime}}, 0\right)-\Omega_{\gamma \gamma} \Omega_{\tau \mu}\left(\gamma^{h^{\prime}}, \tau^{h^{\prime}}, 0\right)<0 \\
\partial \gamma^{h} / \partial \mu \propto \Omega_{\tau \gamma} \Omega_{\tau \mu}\left(\gamma^{h^{\prime}}, \tau^{h^{\prime}}, 0\right)-\Omega_{\tau \tau} \Omega_{\gamma \mu}\left(\gamma^{h^{\prime}}, \tau^{h^{\prime}}, 0\right)>0
\end{array}\right.
$$

from Steps 1 and 2, and because $\Omega_{\tau \gamma}<0, \Omega_{\gamma \gamma}<0$ and $\Omega_{\tau \tau}<0$. This concludes the proof.

Acknowledgements We would like to thank the editor and two anonymous referees for valuable comments and suggestions that greatly improved the paper. The usual disclaimer applies.

Funding Open access funding provided by Università degli Studi di Salerno within the CRUI-CARE Agreement.

Open Access This article is licensed under a Creative Commons Attribution 4.0 International License, which permits use, sharing, adaptation, distribution and reproduction in any medium or format, as long as you give appropriate credit to the original author(s) and the source, provide a link to the Creative Commons licence, and indicate if changes were made. The images or other third party material in this article are included in the article's Creative Commons licence, unless indicated otherwise in a credit line to the material. If material is not included in the article's Creative Commons licence and your intended use is not permitted by statutory regulation or exceeds the permitted use, you will need to obtain permission directly from the copyright holder. To view a copy of this licence, visit http://creativecommons.org/licen ses/by/4.0/. 


\section{References}

Ainslie, G. (1992). Picoeconomics. Cambridge University Press.

Allcott, H., Lockwood, B. B., \& Taubinsky, D. (2019a). Should we tax sugar-sweetened beverages? An overview of theory and evidence. Journal of Economic Perspectives, 33, 202-227.

Allcott, H., Lockwood, B. B., \& Taubinsky, D. (2019b). Regressive sin taxes, with an application to the optimal soda tax. The Quarterly Journal of Economics, 134, 1557-1626.

Allcott, H., \& Taubinsky, D. (2015). Evaluating behaviorally motivated policy: Experimental evidence from the lightbulb market. American Economic Review, 105, 2501-38.

Babor, T., Caetano, R., Casswell, S., Edwards, G., Giesbrecht, N., Graham, K., et al. (2003). Alcohol: No ordinary commodity. Research and public policy. Oxford University Press.

Bénabou, R. (2012). Groupthink: Collective delusions in organizations and markets. Review of Economic Studies, 80, 429-462.

Bénabou, R., \& Tirole, J. (2002). Self-confidence and personal motivation. The Quarterly Journal of Economics, 117, 871-915.

Becker, G. S., \& Murphy, K. M. (1988). A theory of rational addiction. Journal of Political Economy, 96, 675-700.

Chari, V. V., \& Kehoe, P. J. (1999). Optimal fiscal and monetary policy. In J. B. Taylor \& M. Woodford (Eds.), Handbook of macroeconomics, chapter 26 (pp. 1671-1745). Berlin: Elsevier.

Cioffi, C. E., Levitsky, D. A., Pacanowski, C. R., \& Bertz, F. (2015). A nudge in a healthy direction: The effect of nutrition labels on food purchasing behaviors in university dining facilities. Appetite, 92, $7-14$.

Della Vigna, S. (2009). Psychology and economics: Evidence from the field. Journal of Economic Literature, 47, 315-372.

Dillard, A. J., Midboe, A. M., \& Klein, W. M. P. (2009). The dark side of optimism: Unrealistic optimism about problems with alcohol predicts subsequent negative event experiences. Personality and Social Psychology Bulletin, 35, 1540-1550.

Frederick, S., Loewenstein, G., \& O’Donoghue, T. (2002). Time discounting and time preference: A critical review. Journal of Economic Literature, 40, 351-401.

Gervais, S., Heaton, J. B., \& Odean, T. (2003). Overconfidence, investment policy, and executive stock options. Mimeo.

Glaeser, E. L. (2006). Paternalism and psychology. The University of Chicago Law Review, 73(1), 1-21.

Goel, A. M., \& Thakor, A. V. (2008). Overconfidence, CEO selection and corporate governance. Journal of Finance, 53, 2737-2784.

Gruber, J. (2001). Tobacco at the crossroads: The past and future of smoking regulation in the United States. Journal of Economic Perspectives, 15, 193-212.

Gruber, J., \& Koszegi, B. (2001). Is addiction 'rational'? Theory and evidence. Quarterly Journal of Economics, 116, 1261-1305.

Gruber, J., \& Koszegi, B. (2004). Tax incidence when individuals are time-inconsistent: The case of cigarette excise taxes. Journal of Public Economics, 88, 1959-1987.

Gruber, J., \& Mullainathan, S. (2005). Do cigarette taxes make smokers happier? Advances in Economic Analysis and Policy, 5, 1-45.

Guiso, L., \& Paiella, M. (2008). Risk aversion, wealth, and background risk. Journal of the European Economic Association, 6, 1109-1150.

Hsieh, C. R., Yen, L. L., Liu, J. T., \& Lin, C. J. (1996). Smoking, health knowledge, and Anti-smoking campaigns: An empirical study in Taiwan. Journal of Health Economics, 15, 87-104.

Immordino, G., Menichini, A. M. C., \& Romano, M. G. (2019). Taxing and regulating vices. Scandinavian Journal of Economics, 122, 622-647.

Kana, K., \& Tsai, W.D. (2004). Obesity and risk knowledge. Journal of Health Economics, 23, 907-934.

Krosnick, J., Malhotra, N., Mo, C., Bruera, E., Chang, L. C., Pasek, J., \& Thomas, R. K. (2017). Perceptions of health risks of cigarette smoking: A new measure reveals widespread misunderstanding. PLOS ONE, 12, e0182063.

Laibson, D. (1997). Golden eggs and hyperbolic discounting. Quarterly Journal of Economics, 112, 443-477.

Le Bodo, Y., Paquette, M. C., \& De Wals, P. (2016). Taxing soda for public health: A Canadian perspective. Springer. 
Malmendier, U., \& Tate, G. (2005). CEO overconfidence and corporate investment. Journal of Finance, 60, 2661-2700.

Malmendier, U., \& Tate, G. (2008). Who makes Acquisitions? CEO overconfidence and the market's reaction. Journal of Financial Economics, 89, 20-43.

Mariotti, T., Schweizer, N., Szech, N., \& von Wangenheim, J. (2019). Information nudges and self control. n. 18-84, IAST Working Papers, Institute for Advanced Study in Toulouse (IAST).

Moran, A. J., \& Roberto, C. A. (2018). Health warning labels correct parents' misperceptions about sugary drink options. American Journal of Preventive Medicine, 55, e19-27.

O’Donoghue, T., \& Rabin, M. (2003). Studying optimal paternalism, illustrated by a model of sin taxes. American Economic Review, Papers and Proceedings, 93, 186-191.

O’Donoghue, T., \& Rabin, M. (2006). Optimal sin taxes. Journal of Public Economics, 90, 1825-49.

OECD. (2011). Tax administration in OECD and selected non-OECD countries: Comparative information series (2010). OECD Publishing.

Petrakis E., Sartzetakis E. S., Xepapadeas A. (2005). Environmental information provision as a public policy instrument. Contribution to Economic Analysis \& Policy, 4, Article 14.

PricewaterhouseCoopers. (2015). Paying Taxes: The global picture. World Bank Group.

Radcliffe, N. M., \& Klein, W. M. P. (2002). Dispositional, unrealistic and comparative optimism: Differential relations with the knowledge and processing of risk information and beliefs about personal risk. Personality and Social Psychology Bulletin, 28, 836-846.

Roll, R. (1986). The hubris hypothesis of corporate takeovers. Journal of Business, 59, 197-216.

Sartzetakis, E. S., Xepapadeas, A., \& Petrakis, E. (2012). The role of information provision as a policy instrument to supplement environmental taxes. Environmental and Resource Economics, 52, 347-368.

Scheier, M. F., \& Carver, C. S. (1985). Optimism, hoping, and health: Assessment and implications of generalized outcome expectancies. Health Psychology, 4, 219-247.

Thaler, R. H., \& Sunstein, C. R. (2003). Libertarian paternalism. American Economic Review, 93, $175-179$.

Thomas, R., McLellan, J., \& Perera, R. (2013). School-based programmes for preventing smoking. Cochrane Database of Systematic Review, 30, 1616-2040.

Variyam, J.N., \& Cawley, J. (2006). Nutrition labels and obesity. NBER Working Paper Series.

Waters, E. A., Klein, W. M. P., Moser, R. P., Yu, M., Waldron, W. R., McNeel, T. S., \& Freedman, A. N. (2011). Correlates of unrealistic risk beliefs in a nationally representative sample. Journal of Behavioral Medicine, 34, 225-235.

Publisher's Note Springer Nature remains neutral with regard to jurisdictional claims in published maps and institutional affiliations. 